\title{
THE DYNAMICS AND PINNING OF A SPIKE FOR A REACTION-DIFFUSION SYSTEM*
}

\author{
MICHAEL J. WARD ${ }^{\dagger}$, DARRAGH MCINERNEY ${ }^{\ddagger}$, PAUL HOUSTON ${ }^{\S}$, \\ DAVID GAVAGHAN $₫$, AND PHILIP MAINI"
}

\begin{abstract}
The motion of a one-spike solution to a simplified form of the Gierer-Meinhardt activator-inhibitor model is studied in both a one-dimensional and a two-dimensional domain. The pinning effect on the spike motion associated with the presence of spatially varying coefficients in the differential operator, referred to as precursor gradients, is studied in detail. In the one-dimensional case, we derive a differential equation for the trajectory of the spike in the limit $\varepsilon \rightarrow 0$, where $\varepsilon$ is the activator diffusivity. A similar differential equation is derived for the two-dimensional problem in the limit for which $\varepsilon \ll 1$ and $D \gg 1$, where $D$ is the inhibitor diffusivity. A numerical finiteelement method is presented to track the motion of the spike for the full problem in both one and two dimensions. Finally, the numerical results for the spike motion are compared with corresponding asymptotic results for various examples.
\end{abstract}

Key words. spike, Green's function, finite-element method, pinning, Gierer-Meinhardt

AMS subject classifications. 35B25, 35C20, 35P15

\section{PII. S0036139900375112}

1. Introduction. The generation of spatial pattern and form is one of the major unresolved problems in developmental biology. In 1952, Turing [31] showed mathematically that a pair of reacting and diffusing chemicals could evolve from initial near spatially homogeneous states to spatially varying states via the mechanism of local self-enhancement and long range inhibition. He hypothesized that the resultant chemical concentration profiles could serve as prepatterns, providing information for cells, which would differentiate accordingly, forming a spatial pattern. He termed these chemicals "morphogens," and, although morphogens have yet to be unequivocally identified in biological systems, Turing patterns in chemistry are now well documented. (For a review, see Maini, Painter, and Chau [24].)

Since Turing's seminal paper, many reaction-diffusion systems have been proposed for pattern formation. Perhaps the most well-known models are those of activatorinhibitor type proposed by Gierer and Meinhardt [10]. Their models not only generate spatial patterns but also exhibit size regulation, a phenomenon that occurs in many developmental systems such as head development in the Hydra (cf. [10]). In [10] they showed how a reaction-diffusion system could markedly enhance pre-existing shallow spatial gradients, called precursor gradients, resulting in a highly localized (spike-type) pattern for the activator concentration. Their results were then applied

\footnotetext{
*Received by the editors July 13, 2000; accepted for publication (in revised form) August 30, 2001; published electronically April 19, 2002.

http://www.siam.org/journals/siap/62-4/37511.html

${ }^{\dagger}$ Department of Mathematics, University of British Columbia, Vancouver, BC V6T 1Z2, Canada (ward@math.ubc.ca). The research of this author was supported by NSERC grant 81541.

${ }^{\ddagger}$ Mathematical Institute, Oxford University, Oxford OX1 3LB, UK. The research of this author was supported by a Marie Curie Fellowship as part of the Training and Mobility of Researchers (TMR) program administered by the European Commission.

$\S$ Department of Mathematics and Computer Science, University of Leicester, University Road, Leicester LE1 7RH, UK (paul.houston@mcs.le.ac.uk).

`Computing Laboratory, Wolfson Building, Parks Road, Oxford University, Oxford OX1 3QD, UK (david.gavaghan@comlab.ox.ac.uk).

" Mathematical Institute, Oxford University, Oxford OX1 3LB, UK (maini@maths.ox.ac.uk).
} 
to model the head formation in the Hydra. In addition, a precursor gradient in the Gierer-Meinhardt (GM) system was used in the numerical simulations of [15] to model the formation and localization of heart tissue in the Axolotl, a type of salamander. Other applications of precursor gradients in the GM system are discussed in [16] and [14].

From a mathematical viewpoint, precursor gradients are typically modeled by introducing spatial variations in the coefficients in the nonlinear reaction-diffusion system. However, since such systems are difficult to study analytically, the previous work on the GM model with precursor gradients has involved either full numerical simulations (cf. [10], [25], [14], [15], [16]) or else a weakly nonlinear analysis (cf. [17], [18]).

For the simpler case of a scalar singularly perturbed reaction-diffusion equation, there have been many mathematical studies of the effect of spatially inhomogeneous coefficients in different settings. In particular, in the field of superconductivity, a spatially inhomogeneous coefficient is known to induce a pinning phenomena, whereby the dynamics of localized vortex states have new equilibria at certain points determined by properties associated with the spatially inhomogeneous coefficient (see [5] and [23]). In the study [3] of hot-spots arising in the microwave heating of ceramics, a spatially inhomogeneous coefficient, representing an imposed electric field, determines the spatial extent of the hot-spot region. Hot-spot solutions have also been computed for a nonlocal spatially inhomogeneous scalar reaction-diffusion model in [4]. In another problem, the stability of a spike-type solution for a scalar singularly perturbed PDE is studied in [27]. In addition, the existence of multibump solutions for nonlinear Schrödinger-type equations with a potential well is analyzed in [11]. Finally, a mathematical theory for the existence and stability of shock-type solutions to scalar singularly perturbed reaction-diffusion equations with spatially inhomogeneous coefficients is given in [12] and [13]. (See also the references therein.)

The goal of this paper is to study the dynamics of localized solutions to a simplified form of the GM model in a one-dimensional and a two-dimensional domain, while allowing for the effect of precursor gradients. The simplification to the GM model that is made is that we neglect the time dependence associated with the inhibitor field. With this simplification, we are not able to directly model any particular biological application or compare our results on a quantitative basis with those computed numerically in the previous modeling studies mentioned above. Instead, our goal is to develop an asymptotic theory to analyze dynamically the effect of precursor gradients on the simplified GM system for patterns that exhibit strong spatial variations. This analysis is in contrast to the weakly nonlinear analysis of [17] and [18] in one spatial dimension. Mathematically, our analysis extends the previous analytical work on the effect of precursor gradients in scalar problems to an elliptic-parabolic system of PDEs.

Neglecting the dynamics of the inhibitor field, the dimensionless GM model in a one-dimensional domain reduces to the following reaction-diffusion system of activator-inhibitor type (cf. [19]):

$$
\begin{aligned}
a_{t} & =\varepsilon^{2} a_{x x}-[1+V(x)] a+\frac{a^{p}}{h^{q}}, \quad-1<x<1, \quad t>0, \\
0 & =D h_{x x}-\mu(x) h+\varepsilon^{-1} \frac{a^{r}}{h^{s}}, \quad-1<x<1, \quad t>0, \\
a_{x}( \pm 1, t) & =h_{x}( \pm 1, t)=0 .
\end{aligned}
$$




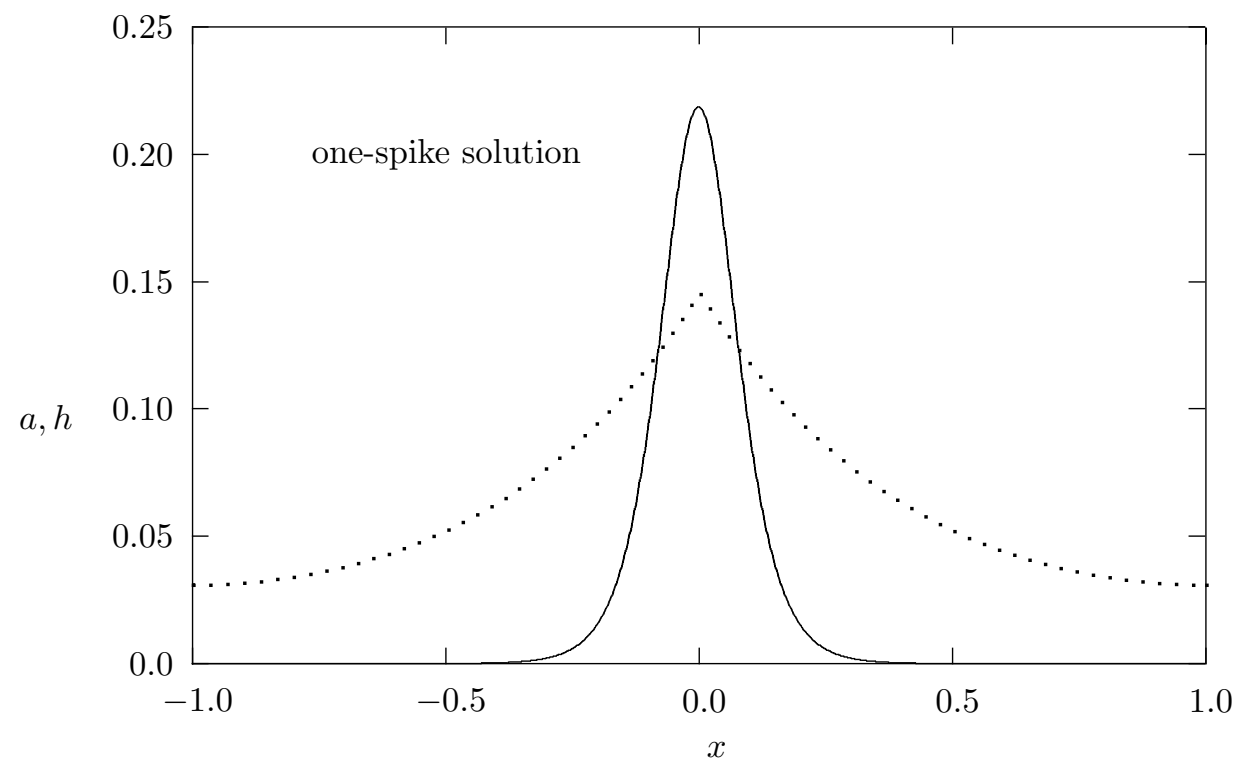

FIG. 1. Plot of a one-spike equilibrium solution to (1.1) computed numerically with $(p, q, r, s)=$ $(2,1,2,0), V \equiv 0, \epsilon=.05, \mu \equiv 1.0$, and $D=.20$. The solid curve is the activator concentration, and the dotted curve is the inhibitor concentration.

Here $a, h, \varepsilon, D>0, \mu(x)>0$, and $V=V(x)>0$ represent the scaled activator concentration, inhibitor concentration, activator diffusivity, inhibitor diffusivity, inhibitor decay rate, and activator decay rate, respectively. The terms $V$ and $\mu$ represent the precursor gradients. The exponents $(p, q, r, s)$ in (1.1) are assumed to satisfy

$$
p>1, \quad q>0, \quad r>0, \quad s \geq 0, \quad \frac{p-1}{q}<\frac{r}{s+1} .
$$

In (1.1) we assume that $\varepsilon \ll 1$ so that the activator diffuses more slowly than does the inhibitor. The analogous problem in a two-dimensional domain is (cf. [19])

$$
\begin{aligned}
a_{t} & =\varepsilon^{2} \triangle a-[1+V(\boldsymbol{x})] a+\frac{a^{p}}{h^{q}}, \quad \boldsymbol{x} \in \Omega, \quad t>0, \\
0 & =D \triangle h-\mu(\boldsymbol{x}) h+\varepsilon^{-2} \frac{a^{r}}{h^{s}}, \quad \boldsymbol{x} \in \Omega, \quad t>0, \\
\partial_{n} a & =\partial_{n} h=0, \quad \boldsymbol{x} \in \partial \Omega .
\end{aligned}
$$

Here $\partial_{n}$ is the outward normal derivative to the boundary, and $\Omega$ is a bounded domain in $\mathcal{R}^{2}$.

For $\varepsilon \ll 1$, many numerical studies of the GM model (1.1) (i.e., [10], [14]) have shown that the solution to (1.1) can have one or more spikes in the activator concentration $a$. These spikes, which represent strong localized deviations from a constant background concentration, have a spatial extent of $O(\varepsilon)$. In Figure 1 we plot a one-spike equilibrium solution to (1.1) for $V \equiv 0, \mu \equiv 1$, and certain other specific parameter values. In this case, an equilibrium one-spike solution is, by symmetry considerations, centered at the midpoint of the interval as shown in Figure 1.

Most of the previous studies for (1.1) and (1.3) have neglected the effect of precursor gradients by taking $V \equiv 0$ and $\mu=1$. Under this assumption, the existence 
of symmetric $k$-spike equilibrium solutions for (1.1) was proved in [30]. The stability properties of these solutions was studied in [21]. In particular, in [21] it was shown that for (1.1) there exist critical values $D_{N}$ of $D$ such that if $D_{N+1}<D<D_{N}$, then a symmetric spike pattern with at most $N$ spikes is stable. Formulae for these critical values $D_{N}$ were calculated explicitly in [21]. In [33], critical values of $D$ characterizing the stability of $N$-spike solutions have also been derived for the two-dimensional problem (1.3). Finally, the dynamics of multispike solutions for (1.1) when $V(x) \equiv 0$ and $\mu=1$ have been studied in [20]. Specifically, a differential-algebraic system of ODEs for the evolution of the centers of the spikes was derived along with criteria predicting the onset of spike collapse events.

The situation is very different when $V(x)$ and $\mu(x)$ are allowed to have spatial variations. These precursor gradients can influence the dynamics, equilibria, and stability of spike solutions. Specifically, it has been observed in previous numerical studies that the precursor gradients can limit the region where spike formation can occur (cf. [14]). As a partial analytical explanation of this observation, we show that the motion of a spike can be pinned to certain points in the domain due to the presence of these gradients. In our analysis, we allow for arbitrary forms for $V(x)$ and $\mu(x)$. However, in our examples of the theory, we take specific forms for these gradients. An exponential function for $\mu(x)$, similar to that used in [14], is chosen in the onedimensional case. A potential well is chosen for $V(x)$ since, based on the analysis of [11] for the nonlinear Schrödinger equation, we might anticipate that a new spike equilibrium located at the minimum of the potential well may be introduced. Other forms for $\mu$ and $V$ are possible, including linear, quadratic, and exponential functions as discussed in [14].

The outline of the paper is as follows. In section 2 we examine the effects of a spatially variable inhibitor decay rate $\mu=\mu(x)>0$ and a potential $V(x)$ on the dynamics and the equilibrium locations of a one-spike solution to the one-dimensional problem (1.1). In particular, we derive an asymptotic differential equation for the center $x_{0}(t)$ of a one-spike solution to (1.1). We show that the exact equilibrium location now depends on certain pointwise values of $V(x)$ and on certain global properties associated with $\mu(x)$ over the domain. In section 3 we treat the two-dimensional problem (1.3). In the recent paper [7] a differential equation for the center of the spike was derived for the special case when $p=2, q=1, V \equiv 0$, and $\mu \equiv 1$. We extend this result using the method of matched asymptotic expansions to derive a similar result for the more general case of arbitrary $p$ and $q$, allowing $V$ and $\mu$ to have a spatial dependence. The analysis is valid when $D \gg-\log \varepsilon$ and $\varepsilon \ll 1$. In section 4 we present a finite-element method to solve the one-dimensional and the two-dimensional problems (1.1) and (1.3) numerically. In sections 5 and 6 we compare the asymptotic results of sections 2 and 3 with corresponding numerical results obtained from the finite-element method of section 4 for the one-dimensional and the two-dimensional problems, respectively. Finally, in section 7 we summarize the results obtained and discuss some open problems.

2. One-spike asymptotic dynamics: The one-dimensional case. In this section we analyze the dynamics of a one-spike solution to (1.1). For finite inhibitor diffusivity $D$, we derive a differential equation determining the location $x_{0}(t)$ of the maximum of the activator concentration for a one-spike solution to (1.1).

In the inner region near the spike we introduce the new variables

$$
y=\varepsilon^{-1}\left[x-x_{0}(\tau)\right], \quad \tilde{h}(y)=h\left(x_{0}+\varepsilon y\right), \quad \tilde{a}(y)=a\left(x_{0}+\varepsilon y\right), \quad \tau=\varepsilon^{2} t .
$$


We then expand the inner solution as

$$
\tilde{h}(y)=\tilde{h}_{0}(y)+\varepsilon \tilde{h}_{1}(y)+\cdots, \quad \tilde{a}(y)=\tilde{a}_{0}(y)+\varepsilon \tilde{a}_{1}(y)+\cdots .
$$

The functions $\tilde{h}_{i}$ and $\tilde{a}_{i}$ will depend parametrically on $\tau$. The spike location is chosen to satisfy $\tilde{a}^{\prime}(0)=0$. Substituting (2.1) into (1.1) and collecting terms that are $O(1)$ as $\varepsilon \rightarrow 0$, we obtain the leading order problems for $\tilde{a}_{0}$ and $\tilde{h}_{0}$,

$$
\begin{aligned}
\tilde{a}_{0}^{\prime \prime}-\left[1+V\left(x_{0}\right)\right] \tilde{a}_{0}+\tilde{a}_{0}^{p} / \tilde{h}_{0}^{q} & =0, \quad-\infty<y<\infty, \\
\tilde{h}_{0}^{\prime \prime} & =0,
\end{aligned}
$$

with $\tilde{a}_{0}^{\prime}(0)=0$. In order to match to the outer solution to be constructed below, we require that $\tilde{h}_{0}$ is independent of $y$. Thus we set $\tilde{h}_{0}=H$, where $H=H(\tau)$ is a function to be determined. We then write the solution to $(2.2 \mathrm{a})$ as

$$
\tilde{a}_{0}(y)=H^{\gamma} u_{c}(y), \quad \text { where } \quad \gamma \equiv q /(p-1) .
$$

Here $u_{c}(y)$, which depends parametrically on $\tau$, is the unique solution to

$$
\begin{gathered}
u_{c}^{\prime \prime}-\left[1+V\left(x_{0}\right)\right] u_{c}+u_{c}^{p}=0, \quad-\infty<y<\infty, \\
u_{c}(0)>0, \quad u_{c}^{\prime}(0)=0, \quad u_{c}(y) \sim \alpha e^{-\beta|y|} \quad \text { as } \quad y \rightarrow \pm \infty
\end{gathered}
$$

for some $\alpha=\alpha\left(x_{0}\right)>0$, where $\beta=\sqrt{1+V\left(x_{0}\right)}$. In the special case for which $p=2$, we can calculate explicitly that

$$
u_{c}(y)=\frac{3}{2}\left[1+V\left(x_{0}\right)\right] \operatorname{sech}^{2}\left(\sqrt{1+V\left(x_{0}\right)} y / 2\right) .
$$

Next we collect the $O(\varepsilon)$ terms in the inner region expansion. In this way, we obtain the problem for $\tilde{a}_{1}$ and $\tilde{h}_{1}$,

$$
\begin{aligned}
& \tilde{a}_{1}^{\prime \prime}-\left[1+V\left(x_{0}\right)\right] \tilde{a}_{1}+\frac{p \tilde{a}_{0}^{p-1}}{\tilde{h}_{0}^{q}} \tilde{a}_{1}=\frac{q \tilde{a}_{0}^{p}}{\tilde{h}_{0}^{q+1}} \tilde{h}_{1}-x_{0}^{\prime} \tilde{a}_{0}^{\prime}+y V^{\prime}\left(x_{0}\right) \tilde{a}_{0}, \quad-\infty<y<\infty, \\
& (2.4 \mathrm{~b}) \quad D \tilde{h}_{1}^{\prime \prime}=-\tilde{a}_{0}^{r} / \tilde{h}_{0}^{s} .
\end{aligned}
$$

Here $x_{0}^{\prime} \equiv d x_{0} / d \tau$. We then write $\tilde{a}_{1}$ as

$$
\tilde{a}_{1}=H^{\gamma} u_{1} .
$$

Substituting (2.2c), (2.5), and $\tilde{h}_{0} \equiv H$ into (2.4), we get

$L\left(u_{1}\right) \equiv u_{1}^{\prime \prime}-\left[1+V\left(x_{0}\right)\right] u_{1}+p u_{c}^{p-1} u_{1}=\frac{q u_{c}^{p}}{H} \tilde{h}_{1}-x_{0}^{\prime} u_{c}^{\prime}+y V^{\prime}\left(x_{0}\right) u_{c}, \quad-\infty<y<\infty$,

$$
D \tilde{h}_{1}^{\prime \prime}=-H^{\gamma r-s} u_{c}^{r} \text {, }
$$

where $u_{1}$ is to decay exponentially as $|y| \rightarrow \infty$. Since $L\left(u_{c}^{\prime}\right)=0$ and $u_{c}^{\prime} \rightarrow 0$ exponentially as $|y| \rightarrow \infty$, the right-hand side of (2.6a) must satisfy the solvability condition that it is orthogonal to $u_{c}^{\prime}$. From this condition, we obtain

$$
\frac{q}{H} \int_{-\infty}^{\infty} u_{c}^{p} u_{c}^{\prime} \tilde{h}_{1} d y+V^{\prime}\left(x_{0}\right) \int_{-\infty}^{\infty} y u_{c} u_{c}^{\prime} d y=x_{0}^{\prime} \int_{-\infty}^{\infty}\left(u_{c}^{\prime}\right)^{2} d y
$$


The second term on the left-hand side of (2.7) is evaluated as

$$
V^{\prime}\left(x_{0}\right) \int_{-\infty}^{\infty} y u_{c} u_{c}^{\prime} d y=-\frac{V^{\prime}\left(x_{0}\right)}{2} \int_{-\infty}^{\infty} u_{c}^{2} d y .
$$

To evaluate the first term on the left-hand side of (2.7), we integrate by parts twice and use the fact that $\tilde{h}_{1}^{\prime \prime}$ and $u_{c}$ are even functions. In this way, we get the differential equation

$$
\begin{aligned}
x_{0}^{\prime} \int_{-\infty}^{\infty}\left(u_{c}^{\prime}\right)^{2} d y= & -\frac{q}{2 H(p+1)} \int_{-\infty}^{\infty} u_{c}^{p+1} d y\left(\lim _{y \rightarrow+\infty} \tilde{h}_{1}^{\prime}+\lim _{y \rightarrow-\infty} \tilde{h}_{1}^{\prime}\right) \\
& -\frac{V^{\prime}\left(x_{0}\right)}{2} \int_{-\infty}^{\infty} u_{c}^{2} d y .
\end{aligned}
$$

Next we analyze the solution in the outer region defined at an $O(1)$ distance away from the center of the spike. In this region, $a$ is exponentially small, and we expand $h$ as $h=h_{0}(x)+o(1)$ as $\varepsilon \rightarrow 0$. Then, from (1.1b), we obtain that $h_{0}$ satisfies

$$
\begin{aligned}
D h_{0}^{\prime \prime}-\mu h_{0} & =-H^{\gamma r-s} b_{r} \delta\left(x-x_{0}\right), \quad-1<x<1, \\
h_{0}^{\prime}( \pm 1) & =0 .
\end{aligned}
$$

Here $b_{r}=b_{r}\left(x_{0}\right)$ is defined by

$$
b_{r} \equiv \int_{-\infty}^{\infty}\left[u_{c}(y)\right]^{r} d y
$$

Solving for $h_{0}$, we have

$$
h_{0}(x)=H^{\gamma r-s} b_{r} G\left(x ; x_{0}\right),
$$

where Green's function $G\left(x ; x_{0}\right)$ satisfies

$$
\begin{aligned}
D G_{x x}-\mu G & =-\delta\left(x-x_{0}\right), \quad-1<x<1, \\
G_{x}\left( \pm 1 ; x_{0}\right) & =0 .
\end{aligned}
$$

To match the outer and inner solutions, we require that

$$
h_{0}\left(x_{0}\right)=H, \quad \lim _{y \rightarrow+\infty} \tilde{h}_{1}^{\prime}+\lim _{y \rightarrow-\infty} \tilde{h}_{1}^{\prime}=h_{0}^{\prime}\left(x_{0+}\right)+h_{0}^{\prime}\left(x_{0-}\right) .
$$

Substituting (2.11) into (2.13), we get

$$
\begin{aligned}
\lim _{y \rightarrow+\infty} \tilde{h}_{1}^{\prime}+\lim _{y \rightarrow-\infty} \tilde{h}_{1}^{\prime} & =\frac{H}{G\left(x_{0} ; x_{0}\right)}\left[G_{x}\left(x_{0+} ; x_{0}\right)+G_{x}\left(x_{0-} ; x_{0}\right)\right], \\
H & =\left[\frac{1}{b_{r} G\left(x_{0} ; x_{0}\right)}\right]^{1 /[\gamma r-(s+1)]} .
\end{aligned}
$$

Finally, substituting (2.14) into (2.2c), (2.9), and (2.11) and letting $\tau=\varepsilon^{2} t$, we obtain the main result of this section.

Proposition 2.1. For $\varepsilon \ll 1$, the dynamics of a one-spike solution to (1.1) is characterized by

$$
\begin{aligned}
& a(x, t) \sim H^{\gamma} u_{c}\left(\varepsilon^{-1}\left[x-x_{0}(t)\right]\right), \\
& h(x, t) \sim H G\left[x ; x_{0}(t)\right] / G\left[x_{0}(t) ; x_{0}(t)\right],
\end{aligned}
$$


where $H=H(t)$, with $t=\varepsilon^{2} \tau$, is given in (2.14b). The spike location $x_{0}(t)$ satisfies the differential equation

$$
\begin{gathered}
\frac{d x_{0}}{d t} \int_{-\infty}^{\infty}\left[u_{c}^{\prime}(y)\right]^{2} d y \sim-\frac{\varepsilon^{2} q}{2(p+1)} \int_{-\infty}^{\infty}\left[u_{c}(y)\right]^{p+1} d y\left(\frac{G_{x}\left(x_{0+} ; x_{0}\right)+G_{x}\left(x_{0-} ; x_{0}\right)}{G\left(x_{0} ; x_{0}\right)}\right) \\
-\frac{\varepsilon^{2}}{2} V^{\prime}\left(x_{0}\right) \int_{-\infty}^{\infty}\left[u_{c}(y)\right]^{2} d y .
\end{gathered}
$$

Notice that when $V\left(x_{0}\right) \neq 0$, the integrals in (2.15c) may also depend on $x_{0}$.

We can calculate the integrals in (2.15c) explicitly using the differential equation (2.3) for $u_{c}$. As shown in Appendix A, we get

$$
\frac{\int_{-\infty}^{\infty}\left[u_{c}(y)\right]^{p+1} d y}{\int_{-\infty}^{\infty}\left[u_{c}^{\prime}(y)\right]^{2} d y}=\frac{2(p+1)}{p-1}, \quad \frac{\int_{-\infty}^{\infty}\left[u_{c}(y)\right]^{2} d y}{\int_{-\infty}^{\infty}\left[u_{c}^{\prime}(y)\right]^{2} d y}=\left(\frac{p+3}{p-1}\right)\left[1+V\left(x_{0}\right)\right]^{-1} .
$$

Substituting (2.16) into (2.15c), we obtain the following result.

Corollary 2.2. For $\varepsilon \ll 1$, the dynamics of a one-spike solution to (1.1) is characterized by

$$
\frac{d x_{0}}{d t} \sim-\frac{\varepsilon^{2} q}{p-1}\left(\frac{G_{x}\left(x_{0+} ; x_{0}\right)+G_{x}\left(x_{0-} ; x_{0}\right)}{G\left(x_{0} ; x_{0}\right)}\right)-\frac{\varepsilon^{2}}{2}\left(\frac{p+3}{p-1}\right) \frac{V^{\prime}\left(x_{0}\right)}{1+V\left(x_{0}\right)} .
$$

We now study the effect of $\mu(x)$ on the dynamics.

2.1. The effect of $\boldsymbol{\mu}(\boldsymbol{x})$. In the special case where $\mu(x)=\mu$ is a positive constant independent of $x$, we can solve (2.12) explicitly for $G\left(x ; x_{0}\right)$ to get

$$
G\left(x ; x_{0}\right)= \begin{cases}A_{0} \cosh [\theta(1+x)] / \cosh \left[\theta\left(1+x_{0}\right)\right], & -1<x<x_{0}, \\ A_{0} \cosh [\theta(1-x)] / \cosh \left[\theta\left(1-x_{0}\right)\right], & x_{0}<x<1,\end{cases}
$$

where

$$
A_{0} \equiv \frac{1}{\sqrt{\mu D}}\left(\tanh \left[\theta\left(1-x_{0}\right)\right]+\tanh \left[\theta\left(1+x_{0}\right)\right]\right)^{-1}, \quad \theta \equiv(\mu / D)^{1 / 2} .
$$

Substituting (2.18) into (2.17), we obtain the following result.

Corollary 2.3. Let $\varepsilon \ll 1$, and let $\mu(x)=\mu$ be a positive constant. Then the differential equation for the spike location is

$$
\frac{d x_{0}}{d t} \sim-\frac{\varepsilon^{2} q \theta}{p-1}\left(\tanh \left[\theta\left(1+x_{0}\right)\right]-\tanh \left[\theta\left(1-x_{0}\right)\right]\right)-\frac{\varepsilon^{2}}{2}\left(\frac{p+3}{p-1}\right) \frac{V^{\prime}\left(x_{0}\right)}{1+V\left(x_{0}\right)} .
$$

Here $\theta \equiv \sqrt{\mu / D}$.

From (2.19) we can determine the qualitative effect of the potential $V(x)$ on the stability of a one-spike solution. If $V$ is convex with a minimum at some point in $[-1,1]$, then there exists a unique equilibrium solution to (2.19), and this equilibrium solution is stable. The situation is more complicated when $V(x)$ is not convex. For instance, suppose that $V(x)$ is a double-well potential of the form $V(x)=\zeta\left(1-x^{2}\right)^{2}$ with $\zeta>0$. In this case, it is easy to see from (2.19) that when

$$
\frac{\zeta}{1+\zeta}>\omega, \quad \text { where } \quad \omega \equiv \frac{q \theta^{2} \operatorname{sech}^{2} \theta}{p+3}
$$


then $x_{0}=0$ is an unstable equilibrium solution to $(2.19)$, and there exists a stable equilibrium solution on each of the subintervals $-1<x<0$ and $0<x<1$. Alternatively, if $\frac{\zeta}{1+\zeta}<\omega$, then $x_{0}=0$ is the only equilibrium solution to (2.19), and it is stable. We conjecture that when a fixed point $x_{0 e}$ of (2.19) is stable, then the steady-state boundary value problem for (1.1) has a stable one-spike solution centered at $x_{0 e}$. Numerical evidence for this conjecture is seen in the numerical experiments $1-5$ in section 5 .

In general, when $\mu$ depends on $x$, we must compute Green's function satisfying (2.12) to determine the dynamics as described in (2.17). However, to illustrate qualitatively the effect of a spatially varying $\mu(x)$, we now derive an approximate differential equation for $x_{0}$ in the limits $D \gg 1$ and $D \ll 1$, with $D$ independent of $\varepsilon$.

In the limit $D \gg 1$, we expand $G$ as

$$
G\left(x ; x_{0}\right)=G_{0}\left(x ; x_{0}\right)+D^{-1} G_{1}\left(x ; x_{0}\right)+O\left(D^{-2}\right) .
$$

Substituting (2.21) into (2.12) and collecting powers of $D^{-1}$, we get

$$
G_{0 x x}=0, \quad G_{1 x x}=\mu G_{0}-\delta\left(x-x_{0}\right),
$$

with $G_{j x}=0$ at $x= \pm 1$ for $j=0,1$. The problem for $G_{1}$ does not have a solution unless $G_{0}$ satisfies a solvability condition. In this way, we calculate that

$$
G_{0}=\left(2 \mu_{a}\right)^{-1}, \quad G_{1 x}=\left(2 \mu_{a}\right)^{-1} \int_{-1}^{x} \mu(y) d y- \begin{cases}0, & -1<x<x_{0}, \\ 1, & x_{0}<x<1 .\end{cases}
$$

Here $\mu_{a}$ is the average of $\mu$ over the interval, defined by

$$
\mu_{a} \equiv \frac{1}{2} \int_{-1}^{1} \mu(x) d x .
$$

Substituting (2.23) into (2.19), we obtain the following result.

Corollary 2.4. For $\varepsilon \ll 1$ and $D \gg 1$, with $D$ independent of $\varepsilon$, the differential equation (2.17) for the spike location reduces to

$$
\frac{d x_{0}}{d t} \sim-\frac{2 \varepsilon^{2} q}{D(p-1)}\left(\int_{-1}^{x_{0}} \mu(y) d y-\mu_{a}\right)-\frac{\varepsilon^{2}}{2}\left(\frac{p+3}{p-1}\right) \frac{V^{\prime}\left(x_{0}\right)}{1+V\left(x_{0}\right)} .
$$

From (2.25) we notice that, when $D \gg 1$, the $O\left(\varepsilon^{2}\right)$ pinning effect associated with the potential $V(x)$ dominates the pinning effect of order $O\left(\varepsilon^{2} / D\right)$ associated with $\mu(x)$.

Alternatively, when $D \ll 1$, we can readily obtain a WKB solution for (2.12) in the form

$$
G\left(x ; x_{0}\right)=\left\{\begin{array}{l}
A_{0} \mu^{-1 / 4} \cosh \left[D^{-1 / 2} \int_{-1}^{x} \sqrt{\mu(s)} d s\right] / \cosh \left[D^{-1 / 2} \int_{-1}^{x_{0}} \sqrt{\mu(s)} d s\right], \\
-1<x<x_{0}, \\
A_{0} \mu^{-1 / 4} \cosh \left[D^{-1 / 2} \int_{x}^{1} \sqrt{\mu(s)} d s\right] / \cosh \left[D^{-1 / 2} \int_{x_{0}}^{1} \sqrt{\mu(s)} d s\right], \\
\quad x_{0}<x<1,
\end{array}\right.
$$

where

$$
A_{0} \equiv \frac{1}{\sqrt{D} \mu^{1 / 4}}\left(\tanh \left[D^{-1 / 2} \int_{x_{0}}^{1} \sqrt{\mu(s)} d s\right]+\tanh \left[D^{-1 / 2} \int_{-1}^{x_{0}} \sqrt{\mu(s)} d s\right]\right)^{-1} .
$$

Substituting (2.26) into (2.19), we obtain the next result. 
Corollary 2.5. For $\varepsilon \ll 1$ and $D \ll 1$, with $D$ independent of $\varepsilon$, the differential equation (2.17) for the spike location reduces to

$$
\begin{aligned}
\frac{d x_{0}}{d t} \sim & -\frac{\varepsilon^{2} q}{p-1} \sqrt{\frac{\mu_{0}}{D}}\left[\tanh \left(D^{-1 / 2} \int_{-1}^{x_{0}} \sqrt{\mu(s)} d s\right)-\tanh \left(D^{-1 / 2} \int_{x_{0}}^{1} \sqrt{\mu(s)} d s\right)\right] \\
& -\frac{\varepsilon^{2}}{2}\left(\frac{p+3}{p-1}\right) \frac{V^{\prime}\left(x_{0}\right)}{1+V\left(x_{0}\right)} .
\end{aligned}
$$

Here we have defined $\mu_{0}=\mu\left(x_{0}\right)$.

From (2.25) and (2.27) we observe that the one-spike dynamics depend on global properties associated with $\mu(x)$ but on pointwise properties associated with $V(x)$. This is intuitively clear since, in the limit $D \gg 1,(1.1)$ can be reduced to a nonlocal PDE referred to as the shadow problem [28], [19]. The spike dynamics for this problem depend nonlocally on $h$ and therefore on $\mu(x)$.

3. One-spike asymptotic dynamics: The two-dimensional case. In this section, we analyze the dynamics of a one-spike solution to (1.3) in the limit $\varepsilon \ll 1$ and $D \gg 1$. The precise range of $D$ with respect to $\varepsilon$ for the validity of the analysis is discussed after Proposition 3.2 below. Our goal is to derive a differential equation for the center $\boldsymbol{x}_{\mathbf{0}}$ of the spike as a function of time. In the limit $\varepsilon \ll 1$ and $D \gg 1$, the solution in the inner region, referred to as the core of the spike, has the leading order asymptotic form

$$
\begin{aligned}
a(\boldsymbol{x}, t) & \sim a_{0}(|\boldsymbol{y}|) \equiv \mathcal{H}^{\gamma} u_{c}(|\boldsymbol{y}|), \\
h(\boldsymbol{x}, t) & \sim \mathcal{H} .
\end{aligned}
$$

Here $\gamma=q /(p-1), \boldsymbol{y}=\varepsilon^{-1}\left(\boldsymbol{x}-\boldsymbol{x}_{\mathbf{0}}\right)$, and $\mathcal{H}$ is a function of $\tau$ to be determined. The radially symmetric function $u_{c}(|\boldsymbol{y}|)$, with $\rho=|\boldsymbol{y}|$, is the unique positive solution of

$$
\begin{aligned}
& u_{c}^{\prime \prime}+\frac{1}{\rho} u_{c}^{\prime}-\left[1+V\left(\boldsymbol{x}_{\mathbf{0}}\right)\right] u_{c}+u_{c}^{p}=0, \quad \rho \geq 0, \\
& u_{c}^{\prime}(0)=0, \quad u_{c} \sim \alpha \rho^{-1 / 2} e^{-\beta \rho}, \quad \text { as } \quad \rho \rightarrow \infty .
\end{aligned}
$$

Here $\alpha=\alpha\left(\boldsymbol{x}_{\mathbf{0}}\right)>0$ is some constant and $\beta=\sqrt{1+V\left(\boldsymbol{x}_{\mathbf{0}}\right)}$. The function $u_{c}$ depends on $\boldsymbol{x}_{\mathbf{0}}$ so that the activator concentration in the core depends on the location of the spike.

In the outer region, away from the core of the spike, $a$ is exponentially small, and so the term $\varepsilon^{-2} a^{r} / h^{s}$ in (1.3b) will be exponentially small except when $\boldsymbol{x}$ approaches $\boldsymbol{x}_{\mathbf{0}}$. In the outer region, this term is asymptotically represented as a multiple of a Dirac mass in the form

$$
\varepsilon^{-2} \frac{a^{r}}{h^{s}} \rightarrow 2 \pi \mathcal{H}^{\gamma r-s} b_{r} \delta\left(\boldsymbol{x}-\boldsymbol{x}_{\mathbf{0}}\right), \quad b_{r} \equiv \int_{0}^{\infty}\left[u_{c}(\rho)\right]^{r} \rho d \rho,
$$

where $u_{c}(\rho)$ satisfies (3.2). Substituting (3.3) into (1.3b), we see that the outer problem for $h$ is

$$
\begin{aligned}
D \triangle h-\mu h+2 \pi \mathcal{H}^{\gamma r-s} b_{r} \delta\left(\boldsymbol{x}-\boldsymbol{x}_{\mathbf{0}}\right) & =0, & & \boldsymbol{x} \in \Omega, \\
\partial_{n} h & =0, & & \boldsymbol{x} \in \partial \Omega .
\end{aligned}
$$


We now solve this problem for $D \gg 1$ by expanding $h$ as

$$
h=h_{0}+\frac{1}{D} h_{1}+\frac{1}{D^{2}} h_{2}+\cdots .
$$

Substituting (3.5) into (3.4) and collecting powers of $D^{-1}$, we obtain

$$
\triangle h_{0}=0, \quad x \in \Omega, \quad \partial_{n} h_{0}=0, \quad x \in \partial \Omega,
$$

and

$$
\begin{aligned}
\triangle h_{1} & =\mu h_{0}-2 \pi \mathcal{H}^{\gamma r-s} b_{r} \delta\left(\boldsymbol{x}-\boldsymbol{x}_{\mathbf{0}}\right), \quad \boldsymbol{x} \in \Omega, \\
\partial_{n} h_{1} & =0, \quad \boldsymbol{x} \in \partial \Omega, \\
\int_{\Omega} \mu h_{1} d \boldsymbol{x} & =0 .
\end{aligned}
$$

This last condition, which arises from a solvability condition applied to the problem for $h_{2}$, ensures that the solution to (3.7) is unique.

The solution to (3.6) depends only on time. To match this solution to the inner solution, we require

$$
h_{0}=\mathcal{H} .
$$

The divergence theorem applied to (3.7) determines $\mathcal{H}$ as

$$
\mathcal{H}=\left(\frac{2 \pi b_{r}}{\int_{\Omega} \mu d \boldsymbol{x}}\right)^{\frac{1}{(1+s)-\gamma r}} .
$$

Then, with this value of $\mathcal{H}$, we write the solution to (3.7) as

$$
h_{1}=\mathcal{H} G\left(\boldsymbol{x} ; \boldsymbol{x}_{\mathbf{0}}\right) \int_{\Omega} \mu d \boldsymbol{x}
$$

where $G$ is the modified Green function satisfying

$$
\begin{aligned}
\triangle G & =\frac{\mu}{\int_{\Omega} \mu d \boldsymbol{x}}-\delta\left(\boldsymbol{x}-\boldsymbol{x}_{\mathbf{0}}\right), \quad \boldsymbol{x} \in \Omega, \\
\partial_{n} G & =0, \quad \boldsymbol{x} \in \partial \Omega, \\
\int_{\Omega} \mu G d \boldsymbol{x} & =0 .
\end{aligned}
$$

The two-term expansion obtained from substituting (3.8) and (3.10) into (3.5) is

$$
h \sim \mathcal{H}\left(1+\frac{G\left(\boldsymbol{x} ; \boldsymbol{x}_{\mathbf{0}}\right)}{D} \int_{\Omega} \mu d \boldsymbol{x}\right) .
$$

The solution to (3.11) can be written as a sum of a singular part and a regular part $R$ as

$$
G\left(\boldsymbol{x} ; \boldsymbol{x}_{\mathbf{0}}\right)=-\frac{1}{2 \pi} \log \left|\boldsymbol{x}-\boldsymbol{x}_{\mathbf{0}}\right|+R\left(\boldsymbol{x} ; \boldsymbol{x}_{\mathbf{0}}\right) .
$$

As $\boldsymbol{x} \rightarrow \boldsymbol{x}_{\mathbf{0}}$, we can expand $R$ in a Taylor series as

$R\left(\boldsymbol{x} ; \boldsymbol{x}_{\mathbf{0}}\right)=R_{0}+\boldsymbol{\nabla} R_{0} \cdot\left(\boldsymbol{x}-\boldsymbol{x}_{\mathbf{0}}\right)+\cdots, \quad R_{0} \equiv R\left(\boldsymbol{x}_{\mathbf{0}} ; \boldsymbol{x}_{\mathbf{0}}\right),\left.\quad \boldsymbol{\nabla} R_{0} \equiv \boldsymbol{\nabla} R\left(\boldsymbol{x} ; \boldsymbol{x}_{\mathbf{0}}\right)\right|_{\boldsymbol{x}=\boldsymbol{x}_{\mathbf{0}}}$. 
Therefore, the two-term expansion (3.12) for $h$ has the following behavior as $\boldsymbol{x} \rightarrow \boldsymbol{x}_{\mathbf{0}}$ :

$$
h \sim \mathcal{H}\left[1+\frac{1}{D} \int_{\Omega} \mu d \boldsymbol{x}\left(-\frac{1}{2 \pi} \log \left|\boldsymbol{x}-\boldsymbol{x}_{\mathbf{0}}\right|+R_{0}+\boldsymbol{\nabla} R_{0} \cdot\left(\boldsymbol{x}-\boldsymbol{x}_{\mathbf{0}}\right)\right)\right] .
$$

We now construct the inner expansion and derive the differential equation for $\boldsymbol{x}_{\mathbf{0}}$ in two limiting regimes of $D$ and $\varepsilon$.

3.1. The effect of the potential. The form (3.15) suggests that in the inner (core) region the change in $h$ from a constant value will be of the order $O(-\log \varepsilon / D)$. In this region, the perturbation to $a$ induced by the gradient of the potential is of the order $O(\varepsilon)$. Hence, as $\varepsilon \rightarrow 0$, we will assume at present that $D$ is large enough to satisfy

$$
D \gg-\varepsilon^{-1} \log \varepsilon .
$$

This restriction on $D$ can be weakened substantially as discussed following (3.39) below. Under (3.16) we can expand $a$ and $h$ in the core region as

$$
a(\boldsymbol{x}, t)=\mathcal{H}^{\gamma} u_{c}\left[\varepsilon^{-1}\left(\boldsymbol{x}-\boldsymbol{x}_{\mathbf{0}}(\tau)\right)\right]+\varepsilon \tilde{a}_{1}+\cdots, \quad h=\mathcal{H}+O(\varepsilon),
$$

where $\tau=\varepsilon^{2} t$. Substituting (3.17) into (1.3a) and writing $\tilde{a}_{1}$ as

$$
\tilde{a}_{1}=\mathcal{H}^{\gamma} u_{1}
$$

we get that $u_{1}$ satisfies

$$
L\left(u_{1}\right) \equiv \triangle u_{1}-\left[1+V\left(\boldsymbol{x}_{\mathbf{0}}\right)\right] u_{1}+p u_{c}^{p-1} u_{1}=\nabla V\left(\boldsymbol{x}_{\mathbf{0}}\right) \cdot \boldsymbol{y} u_{c}-u_{c}{ }^{\prime} \frac{\boldsymbol{x}_{\mathbf{0}}{ }^{\prime} \cdot \boldsymbol{y}}{|\boldsymbol{y}|} .
$$

Here $\boldsymbol{x}_{\mathbf{0}}{ }^{\prime} \equiv d \boldsymbol{x}_{\mathbf{0}} / d \tau$ and $u_{1} \rightarrow 0$ is to tend exponentially to zero as $|\boldsymbol{y}| \rightarrow \infty$. Since $L\left(\partial_{y_{j}} u_{c}\right)=0$ for $j=1,2$, this problem for $u_{1}$ has a solution only when the right-hand side of (3.19) is orthogonal to $\partial_{y_{j}} u_{c}$ for $j=1,2$. Hence the solvability condition for (3.19) is

$$
\boldsymbol{x}_{\mathbf{0}}{ }^{\prime} \cdot \int_{\mathcal{R}^{2}} \frac{\boldsymbol{y}}{|\boldsymbol{y}|} u_{c}^{\prime} \partial_{y_{j}} u_{c} d \boldsymbol{y}=\nabla V\left(\boldsymbol{x}_{\mathbf{0}}\right) \cdot \int_{\mathcal{R}^{2}} \boldsymbol{y} u_{c} \partial_{y_{j}} u_{c} d \boldsymbol{y}
$$

for $j=1,2$. Upon integrating by parts and using symmetry, we can readily derive that

$$
\begin{aligned}
\int_{\mathcal{R}^{2}} y_{k} u_{c} \partial_{y_{j}} u_{c} d \boldsymbol{y} & =-\pi \int_{0}^{\infty}\left[u_{c}(\rho)\right]^{2} \rho d \rho \delta_{j, k}, \\
\int_{\mathcal{R}^{2}} \frac{y_{k}}{|\boldsymbol{y}|} u_{c}^{\prime} \partial_{y_{j}} u_{c} d \boldsymbol{y} & =\pi \int_{0}^{\infty}\left[u_{c}^{\prime}(\rho)\right]^{2} \rho d \rho \delta_{j, k} .
\end{aligned}
$$

Here $\delta_{j, k}$ is the usual Kronecker symbol. Substituting (3.21) into (3.20), we obtain the following main result.

Proposition 3.1. For $\varepsilon \ll 1$ and $D$ satisfying (3.16), the dynamics of a onespike solution to (1.3) in the core are characterized by

$$
\begin{aligned}
a(x, t) & \sim \mathcal{H}^{\gamma} u_{c}\left[\varepsilon^{-1}\left|\boldsymbol{x}-\boldsymbol{x}_{\mathbf{0}}(t)\right|\right]+O(\varepsilon), \\
h(x, t) & \sim \mathcal{H}+o(\varepsilon),
\end{aligned}
$$


where $u_{c}$ and $\mathcal{H}$ are defined in (3.2) and (3.9), respectively. The differential equation for the center $\boldsymbol{x}_{\mathbf{0}}$ of the spike is

$$
\frac{d \boldsymbol{x}_{\mathbf{0}}}{d t} \sim-\varepsilon^{2} \boldsymbol{\nabla} V\left(\boldsymbol{x}_{\mathbf{0}}\right)\left(\frac{\int_{0}^{\infty}\left[u_{c}(\rho)\right]^{2} \rho d \rho}{\int_{0}^{\infty}\left[u_{c}^{\prime}(\rho)\right]^{2} \rho d \rho}\right) .
$$

From (3.2) it is clear that $u_{c}$ depends on $\boldsymbol{x}_{\mathbf{0}}$. Thus the integrals in (3.9) for $H$ and in (3.22c) for the dynamics of $\boldsymbol{x}_{\mathbf{0}}$ also depend on $\boldsymbol{x}_{\mathbf{0}}$. To explicitly show this dependence, we introduce the new variables $\eta$ and $w_{c}$ defined by

$$
\rho=\left[1+V\left(\boldsymbol{x}_{\mathbf{0}}\right)\right]^{-1 / 2} \eta, \quad u_{c}=\left[1+V\left(\boldsymbol{x}_{\mathbf{0}}\right)\right]^{1 /(p-1)} w_{c} .
$$

Substituting (3.23) into (3.2), we find that $w_{c}(\eta)$ satisfies

$$
\begin{array}{rlrl}
w_{c}^{\prime \prime}+\frac{1}{\eta} w_{c}^{\prime}-w_{c}+w_{c}^{p} & =0, \quad \eta & \geq 0, \\
w_{c}^{\prime}(0) & =0, \quad w_{c} \sim \alpha \eta^{-1 / 2} e^{-\eta} \quad \text { as } \quad \eta \rightarrow \infty .
\end{array}
$$

In terms of these variables, (3.9) becomes

$$
\mathcal{H}^{1+s-\gamma r}=C\left[1+V\left(x_{0}\right)\right]^{-1+r /(p-1)}, \quad C \equiv \frac{2 \pi \int_{0}^{\infty} w_{c}^{r} \eta d \eta}{\int_{\Omega} \mu d \boldsymbol{x}},
$$

where $C$ is independent of $\tau$. In addition, the differential equation (3.22c) becomes

$$
\frac{d \boldsymbol{x}_{\mathbf{0}}}{d t} \sim-\varepsilon^{2} b \frac{\nabla V\left(\boldsymbol{x}_{\mathbf{0}}\right)}{1+V\left(\boldsymbol{x}_{\mathbf{0}}\right)}, \quad b \equiv \frac{\int_{0}^{\infty}\left[w_{c}(\eta)\right]^{2} \eta d \eta}{\int_{0}^{\infty}\left[w_{c}^{\prime}(\eta)\right]^{2} \eta d \eta},
$$

where $b$ is independent of $\boldsymbol{x}_{\mathbf{0}}$. In Appendix B, we calculate $b$ as $b=2 /(p-1)$. Then (3.22c) can be written compactly as the gradient flow

$$
\frac{d \boldsymbol{x}_{\mathbf{0}}}{d t} \sim-\frac{2 \varepsilon^{2}}{(p-1)} \boldsymbol{\nabla} W\left(\boldsymbol{x}_{\mathbf{0}}\right), \quad \text { where } \quad W\left(\boldsymbol{x}_{\mathbf{0}}\right) \equiv \log \left[1+V\left(\boldsymbol{x}_{\mathbf{0}}\right)\right] .
$$

From (3.27) we observe that stable equilibria for the spike are located at points where the potential $V(\boldsymbol{x})$ has a local minimum. In addition, the motion of the spike is orthogonal to level curves of the potential $W\left(\boldsymbol{x}_{\mathbf{0}}\right)$ and $d W\left(\boldsymbol{x}_{\mathbf{0}}\right) / d t<0$ except at critical points of $W$.

3.2. The regular part of Green's function. In the derivation below, we assume that $V(\boldsymbol{x}) \equiv 0$ in (1.3). Our expansion parameter is taken as $\varepsilon / D$, and we will use $D \gg 1$ to simplify some of the terms that arise in the expansion. In the core region, we begin by introducing new variables

$$
\tilde{a}(\boldsymbol{y})=a\left(\boldsymbol{x}_{\mathbf{0}}+\varepsilon \boldsymbol{y}, t\right), \quad \tilde{h}(\boldsymbol{y})=h\left(\boldsymbol{x}_{\mathbf{0}}+\varepsilon \boldsymbol{y}, t\right), \quad \boldsymbol{y}=\varepsilon^{-1}\left[\boldsymbol{x}-\boldsymbol{x}_{\mathbf{0}}(\tau)\right],
$$

where $\tau$ is a slow time scale. We then expand $\tilde{a}$ and $\tilde{h}$ as

$$
\tilde{a}(\boldsymbol{y})=\tilde{a}_{0}(|\boldsymbol{y}|)+\frac{\varepsilon}{D} \tilde{a}_{1}(\boldsymbol{y})+\cdots, \quad \tilde{h}(\boldsymbol{y})=\tilde{h}_{0}(|\boldsymbol{y}|)+\frac{\varepsilon}{D} \tilde{h}_{1}(\boldsymbol{y})+\cdots .
$$

Here $\tilde{a}_{0}$ and $\tilde{h}_{0}$ are radially symmetric and are at most $O(-\log \varepsilon)$ as $\varepsilon \rightarrow 0$. The functions $\tilde{a}_{1}$ and $\tilde{h}_{1}$ are not radially symmetric. A nontrivial solvability condition will 
arise at the problem for $\tilde{a}_{1}$ and $\tilde{h}_{1}$. This forces us to introduce the slow time scale $\tau$ defined by

$$
\tau=\frac{\varepsilon^{2}}{D} t
$$

Substituting (3.29) and (3.30) into (1.3a) and (1.3b) and collecting powers of $\varepsilon / D$, we obtain the problems

$$
\begin{aligned}
\triangle \tilde{a}_{0}-\tilde{a}_{0}+\frac{\tilde{a}_{0}^{p}}{\tilde{h}_{0}^{q}} & =0, & & |\boldsymbol{y}| \geq 0, \\
\triangle \tilde{h}_{0} & =-\frac{\tilde{a}_{0}^{r}}{D \tilde{h}_{0}^{s}}, & & |\boldsymbol{y}| \geq 0,
\end{aligned}
$$

and

$$
\begin{aligned}
\triangle \tilde{a}_{1}-\tilde{a}_{1}+\frac{p \tilde{a}_{0}^{p-1}}{\tilde{h}_{0}^{q}} \tilde{a}_{1} & =\frac{q \tilde{a}_{0}^{p}}{\tilde{h}_{0}^{q+1}} \tilde{h}_{1}-\tilde{a}_{0}^{\prime} \frac{\boldsymbol{x}_{\mathbf{0}}{ }^{\prime} \cdot \boldsymbol{y}}{|\boldsymbol{y}|}, & \boldsymbol{y} \in \mathcal{R}^{2}, \\
\triangle \tilde{h}_{1} & =-\frac{1}{D}\left[\frac{r \tilde{a}_{0}^{r-1}}{\tilde{h}_{0}^{s}} \tilde{a}_{1}-\frac{s \tilde{a}_{0}^{r}}{\tilde{h}_{0}^{s+1}} \tilde{h}_{1}\right], & \boldsymbol{y} \in \mathcal{R}^{2} .
\end{aligned}
$$

Here $\boldsymbol{x}_{\mathbf{0}}{ }^{\prime} \equiv d \boldsymbol{x}_{\mathbf{0}} / d \tau$. The matching conditions as $|\boldsymbol{y}| \rightarrow \infty$ obtained from (3.15) are

$$
\begin{aligned}
\tilde{h}_{0} & \sim \mathcal{H}\left[1+\frac{1}{D} \int_{\Omega} \mu d \boldsymbol{x}\left(-\frac{1}{2 \pi} \log |\boldsymbol{y}|-\frac{1}{2 \pi} \log \varepsilon+R_{0}\right)\right] \quad \text { as } \quad|\boldsymbol{y}| \rightarrow \infty, \\
\tilde{h}_{1} & \sim \mathcal{H} \int_{\Omega} \mu d \boldsymbol{x}\left(\boldsymbol{\nabla} R_{0} \cdot \boldsymbol{y}\right) \quad \text { as } \quad|\boldsymbol{y}| \rightarrow \infty
\end{aligned}
$$

When $D \gg 1$, the leading order problems for $\tilde{a}_{0}$ and $\tilde{h}_{0}$ become decoupled. In this limit, there is a unique solution to (3.31) with the following leading order expansion in $D$ :

$$
\tilde{a}_{0} \sim \mathcal{H}^{\gamma} w_{c}(|\boldsymbol{y}|)+O\left(D^{-1}\right), \quad \tilde{h}_{0} \sim \mathcal{H}+O\left(D^{-1}\right) .
$$

Here $\mathcal{H}$ and $w_{c}$ were defined in (3.9) and (3.24), respectively. In addition, in the limit $D \gg 1$, the solvability condition for (3.32a) is that the right-hand side of (3.32a) must be orthogonal to $\partial_{y_{j}} w_{c}$ for $j=1,2$. This condition yields a differential equation for $x_{0}$ in the form

$$
\boldsymbol{x}_{\mathbf{0}}{ }^{\prime} \cdot \int_{\mathcal{R}^{2}} \frac{\boldsymbol{y}}{|\boldsymbol{y}|} \tilde{a}_{0}^{\prime} \partial_{y_{j}} w_{c} d \boldsymbol{y}=\int_{\mathcal{R}^{2}} \frac{q \tilde{a}_{0}^{p}}{\tilde{h}_{0}^{q+1}} \tilde{h}_{1} \partial_{y_{j}} w_{c} d \boldsymbol{y}
$$

To derive an explicit differential equation, we substitute (3.34) into (3.35) to get

$$
\boldsymbol{x}_{\mathbf{0}}{ }^{\prime} \cdot \int_{\mathcal{R}^{2}} \frac{\boldsymbol{y}}{|\boldsymbol{y}|} w_{c}^{\prime} \partial_{y_{j}} w_{c} d \boldsymbol{y}=\frac{q}{(p+1) \mathcal{H}} \int_{\mathcal{R}^{2}} \tilde{h}_{1} \partial_{y_{j}}\left(w_{c}^{p+1}\right) d \boldsymbol{y}, \quad j=1,2 .
$$

The integral term on the left-hand side of (3.36) was evaluated in (3.21). To evaluate the integral term on the right-hand side of (3.36), we integrate by parts twice and use both symmetry and the asymptotic boundary condition (3.33b) as $\boldsymbol{y} \rightarrow \infty$ to get

$$
\int_{\mathcal{R}^{2}} \tilde{h}_{1} \boldsymbol{\nabla}\left(w_{c}^{p+1}\right) d \boldsymbol{y}=-\int_{\mathcal{R}^{2}}\left(w_{c}^{p+1}\right) \nabla \tilde{h}_{1} d \boldsymbol{y} \sim-\mathcal{H} \boldsymbol{\nabla} R_{0} \int_{\Omega} \mu d \boldsymbol{x} \int_{\mathcal{R}^{2}} w_{c}^{p+1} d \boldsymbol{y} .
$$

Substituting (3.37) and (3.21) into (3.36), we obtain the following result. 
Proposition 3.2. Consider a one-spike solution to (1.3) when $V(\boldsymbol{x})=0, \varepsilon \ll 1$, and $D \gg 1$. Then the solution to (1.3) in the core is given by (3.34). The motion of the center of the spike satisfies

$$
\frac{d \boldsymbol{x}_{\mathbf{0}}}{d t} \sim-\frac{2 q \varepsilon^{2}}{D(p+1)} \nabla R_{0} \int_{\Omega} \mu d \boldsymbol{x}\left(\frac{\int_{0}^{\infty}\left[w_{c}(\rho)\right]^{p+1} \rho d \rho}{\int_{0}^{\infty}\left[w_{c}^{\prime}(\rho)\right]^{2} \rho d \rho}\right) .
$$

Here $\boldsymbol{\nabla} R_{0}$ is the gradient of the regular part of Green's function defined in (3.14).

Setting $\mu=1, p=2$, and $q=1$ in (3.38), we obtain the result derived previously in [7] using a different method.

As shown in Appendix B, we can calculate the integral appearing in (3.38) exactly as

$$
\frac{\int_{0}^{\infty}\left[w_{c}(\rho)\right]^{p+1} \rho d \rho}{\int_{0}^{\infty}\left[w_{c}^{\prime}(\rho)\right]^{2} \rho d \rho}=\frac{p+1}{p-1}
$$

There are two important remarks. The first observation is that the differential equation (3.38) derived when $V(\boldsymbol{x}) \equiv 0$ predicts a motion on a time scale of $O\left(\varepsilon^{2} / D\right)$, whereas (3.22c) predicts a motion on a faster time scale of $O\left(\varepsilon^{2}\right)$. Therefore, when $D \gg 1$, the pinning effect induced by the potential dominates the dynamics. The second observation concerns the range of validity of the results (3.22c) and (3.38) with respect to $D$ as $\varepsilon \rightarrow 0$. For the validity of Proposition 3.2, we require that $D \gg-\log \varepsilon$ to ensure that $\tilde{h}_{0}=\mathcal{H}+o(1)$ in the core region and that the leading order problems for $\tilde{a}_{0}$ and $\tilde{h}_{0}$ decouple. With this decoupling that occurs for $D \gg-\log \varepsilon$, the problem for $\tilde{a}_{1}$ is self-adjoint, and the solution to the homogeneous form for (3.32a) is simply $\partial_{y_{j}} w_{c}$.

Similarly, the result (3.22c) for the spike motion in the presence of the potential $V(x)$ is valid for $D \gg-\log \varepsilon$. This condition again ensures that the leading order problems for $a$ and $h$ in the core are decoupled and that the functions $\partial_{y_{j}} u_{c}$ for $j=1,2$ can be used for the solvability condition. However, when $D$ is asymptotically smaller than the estimate given in (3.16), we must modify the inner expansion (3.17) for $a$ and $h$ by inserting intermediate terms of lower order than the $O(\varepsilon)$ terms. The asymptotic matching condition (3.15) ensures that these new terms are radially symmetric functions. Thus they give rise only to trivial solvability conditions. A nontrivial solvability condition arises only from the $O(\varepsilon)$ term in the inner expansion for $a$, which does not have radial symmetry.

3.3. Calculating the regular part of Green's function. We decompose the solution to (3.11) in the form

$$
G\left(\boldsymbol{x} ; \boldsymbol{x}_{\mathbf{0}}\right)=g_{p}(\boldsymbol{x})+g\left(\boldsymbol{x} ; \boldsymbol{x}_{\mathbf{0}}\right)+c\left(\boldsymbol{x}_{\mathbf{0}}\right) .
$$

Here $g_{p}$ and $g$ are any solutions to

$$
\begin{array}{rlrl}
\triangle g_{p} & =\frac{\mu}{\int_{\Omega} \mu d \boldsymbol{x}}, & & \boldsymbol{x} \in \Omega, \\
\partial_{n} g_{p} & =1 / L, & \boldsymbol{x} \in \partial \Omega,
\end{array}
$$

and

$$
\begin{aligned}
\triangle g & =-\delta\left(\boldsymbol{x}-\boldsymbol{x}_{\mathbf{0}}\right), & & \boldsymbol{x} \in \Omega, \\
\partial_{n} g & =-1 / L, & & \boldsymbol{x} \in \partial \Omega .
\end{aligned}
$$


Here $L$ is the perimeter of $\partial D$. To satisfy the condition (3.11c), we chose the constant $c$ in (3.40) as

$$
c \int_{\Omega} \mu d \boldsymbol{x}=-\int_{\Omega} \mu\left(g_{p}+g\right) d \boldsymbol{x} .
$$

In terms of this decomposition, the function $R$ in (3.13) is given by

$$
R\left(\boldsymbol{x} ; \boldsymbol{x}_{\mathbf{0}}\right)=g_{p}(\boldsymbol{x})+\left(g\left(\boldsymbol{x} ; \boldsymbol{x}_{\mathbf{0}}\right)+\frac{1}{2 \pi} \log \left|\boldsymbol{x}-\boldsymbol{x}_{\mathbf{0}}\right|\right)+c\left(\boldsymbol{x}_{\mathbf{0}}\right) .
$$

As an example, consider a circular domain of radius one with $\mu=\mu(|\boldsymbol{x}|)$. For this case, where $L=2 \pi$, we identify points in the circle as complex numbers and then calculate $g$ explicitly from (3.42) as

$$
g\left(\boldsymbol{x} ; \boldsymbol{x}_{\mathbf{0}}\right)=-\frac{1}{2 \pi} \log \left|\boldsymbol{x}-\boldsymbol{x}_{\mathbf{0}}\right|-\frac{1}{2 \pi} \log \left|\boldsymbol{x}_{\mathbf{0}}\right|-\frac{1}{2 \pi} \log \left|\boldsymbol{x}-1 / \overline{\boldsymbol{x}}_{\mathbf{0}}\right| .
$$

Here $\overline{\boldsymbol{x}}_{\mathbf{0}}$ denotes the complex conjugate of $\boldsymbol{x}_{\mathbf{0}}$. From (3.41) we determine $g_{p}$ as

$$
g_{p}=\frac{1}{2 \pi \mu_{a}} \int_{0}^{r} \frac{1}{s}\left(\int_{0}^{s} \eta \mu(\eta) d \eta\right) d s, \quad \mu_{a} \equiv \int_{0}^{1} r \mu(r) d r .
$$

Substituting (3.45) and (3.46) into (3.44), we can calculate the gradient of $R$ at $\boldsymbol{x}_{\mathbf{0}}$ as

$$
\left.\boldsymbol{\nabla} R_{0} \equiv \nabla R\left(\boldsymbol{x} ; \boldsymbol{x}_{\mathbf{0}}\right)\right|_{\boldsymbol{x}=\boldsymbol{x}_{\mathbf{0}}}=\frac{1}{2 \pi}\left[\frac{1}{1-\left|\boldsymbol{x}_{\mathbf{0}}\right|^{2}}+\frac{1}{\mu_{a}\left|\boldsymbol{x}_{\mathbf{0}}\right|^{2}} \int_{0}^{\left|\boldsymbol{x}_{\mathbf{0}}\right|} s \mu(s) d s\right] \boldsymbol{x}_{\mathbf{0}} .
$$

Substituting (3.47) into (3.38) and using (3.39), we obtain the following result.

Corollary 3.3. Under the conditions of Proposition 3.2, let $\mu=\mu(|\boldsymbol{x}|)$, and suppose that $\Omega$ is a circular domain of radius one. Then the motion of the center of the spike satisfies

$$
\frac{d \boldsymbol{x}_{\mathbf{0}}}{d t} \sim-\varepsilon^{2} \kappa \mu_{a}\left[\frac{1}{1-\left|\boldsymbol{x}_{\mathbf{0}}\right|^{2}}+\frac{1}{\mu_{a}\left|\boldsymbol{x}_{\mathbf{0}}\right|^{2}} \int_{0}^{\left|\boldsymbol{x}_{\mathbf{0}}\right|} s \mu(s) d s\right] \boldsymbol{x}_{\mathbf{0}},
$$

where $\mu_{a}$ is defined in (3.46) and

$$
\kappa \equiv \frac{2 q}{D(p-1)} .
$$

From (3.48), it follows that the spike will tend to the origin as $t \rightarrow \infty$. A differential equation for the distance from the center of the spike to the origin can be obtained by taking the dot product of (3.48a) with $\boldsymbol{x}_{\mathbf{0}}$. As an example, suppose that $\mu \equiv 1$. Then, upon defining $\xi=\xi(t)$ by $\xi=\left|\boldsymbol{x}_{\mathbf{0}}\right|^{2}$, we obtain from (3.48a) that

$$
\frac{d \xi}{d t} \sim-\varepsilon^{2} \kappa\left(\frac{2-\xi}{1-\xi}\right) \xi
$$

Upon integrating this differential equation, we obtain the next result.

Corollary 3.4. Let $\mu=1, V \equiv 0$, and $\Omega$ be the unit circle. Suppose that the spike is initially centered at $\boldsymbol{x}_{\mathbf{0}}(0) \in \Omega$. Then, when $\varepsilon \ll 1$ and $D \gg 1$, the distance from the spike to the origin at later times is given by

$$
\left|\boldsymbol{x}_{\mathbf{0}}(t)\right| \sim\left(1-\left[1-\beta e^{-2 \varepsilon^{2} \kappa t}\right]^{1 / 2}\right)^{1 / 2}, \quad \text { where } \quad \beta \equiv 1-\left(1-\left|\boldsymbol{x}_{\mathbf{0}}(0)\right|^{2}\right)^{2} .
$$

Here $\kappa$ is defined in (3.48b). 
4. Finite element discretization. In this section, we outline the numerical method used to compute solutions to (1.3). For simplicity, we write (1.3) in the compact form

$$
M \mathbf{u}_{t}=\nabla \cdot(K \nabla \mathbf{u})+\mathbf{f}(\mathbf{u}, \boldsymbol{x})
$$

where $\mathbf{u}=(u, v)$ and

$$
\begin{aligned}
M & \equiv\left(\begin{array}{cc}
1 & 0 \\
0 & 0
\end{array}\right), \\
K & \equiv\left(\begin{array}{cc}
\epsilon^{2} & 0 \\
0 & D
\end{array}\right), \quad \text { and } \\
\mathbf{f}(\mathbf{u}, \boldsymbol{x}) & \equiv\left(\begin{array}{c}
-[1+V(\boldsymbol{x})] u+u^{p} / v^{q} \\
-\mu v+\epsilon^{-2} u^{r} / v^{s}
\end{array}\right) .
\end{aligned}
$$

Before we define the finite-element discretization of (4.1a), let us first introduce some notation. Let $0=t_{0}<t_{1}<\cdots<t_{N}=T$ be a subdivision of $[0, T]$ with corresponding time steps $k_{n}=t_{n}-t_{n-1}$. For each $n, 0 \leq n \leq N$, let $\mathcal{T}_{n}=\{\kappa\}$ be a finiteelement partition of $\Omega$ into shape regular simplices $\kappa$. For $p \in \mathbb{N}$, we define the finite-element space

$$
S_{h_{n}}=\left\{w \in C(\Omega):\left.w\right|_{\kappa} \in \mathcal{P}_{p}(\kappa) \forall \kappa \in \mathcal{T}_{n}\right\}
$$

for $n=1, \ldots, N$, where $\mathcal{P}_{p}(\kappa)$ denotes the space of polynomials of degree at most $p$ over $\kappa$.

The construction of the finite-element method involves writing the problem (4.1a) in the following weak form: find $\mathbf{u}(t)$ such that

$$
\begin{aligned}
\left(M \mathbf{u}_{t}(\cdot, t), \mathbf{w}\right) & =-(K \nabla \mathbf{u}(\cdot, t), \nabla \mathbf{w})+(\mathbf{f}(\mathbf{u}(\cdot, t), \cdot), \mathbf{w}) & & \forall \mathbf{w} \in \mathcal{V}, \\
(\mathbf{u}(\cdot, 0), \mathbf{w}) & =\left(\mathbf{u}_{0}, \mathbf{w}\right) & & \forall \mathbf{w} \in \mathcal{V} .
\end{aligned}
$$

Here $(\cdot, \cdot)$ denotes the $L_{2}(\Omega)$ inner product, $\mathcal{V}=\left(H^{1}(\Omega)\right)^{2}$, and $H^{1}(\Omega)$ denotes the usual Hilbertian Sobolev space. The time-discretization involves approximating the derivative $\mathbf{u}_{t}$ by a divided difference operator. The simplest appropriate discretization is the backward Euler method, giving for $n=1, \ldots, N$

$$
\begin{array}{rlrl}
\left(M \frac{\mathbf{u}\left(\cdot, t_{n}\right)-\mathbf{u}\left(\cdot, t_{n-1}\right)}{k_{n}}, \mathbf{w}\right) & =-\left(K \nabla \mathbf{u}\left(\cdot, t_{n}\right), \nabla \mathbf{w}\right)+\left(\mathbf{f}\left(\mathbf{u}\left(\cdot, t_{n}\right), \cdot\right), \mathbf{w}\right) & & \forall \mathbf{w} \in \mathcal{V}, \\
(4.4 \mathrm{~b}) & & \forall \mathbf{w} \in \mathcal{V} .
\end{array}
$$

If we now define $\mathbf{u}_{h}^{n}=\left(u_{h}^{n}, v_{h}^{n}\right)$ to be the Galerkin finite-element approximation to $\mathbf{u}\left(\cdot, t_{n}\right)$ at time $t_{n}$, then applying the finite-element method to (4.4) yields the following formulation. Find $\mathbf{u}_{h}^{n} \in\left(S_{h_{n}}\right)^{2}$ for $1 \leq n \leq N$ such that

$$
\begin{aligned}
\left(M \frac{\mathbf{u}_{h}^{n}-\mathbf{u}_{h}^{n-1}}{k_{n}}, \mathbf{w}\right) & =-\left(K \nabla \mathbf{u}_{h}^{n}, \nabla \mathbf{w}\right)+\left(\mathbf{f}\left(\mathbf{u}_{h}^{n}, \boldsymbol{x}_{n}\right), \mathbf{w}\right) & & \forall \mathbf{w} \in\left(S_{h_{n}}\right)^{2}, \\
\left(\mathbf{u}_{h}^{0}, \mathbf{w}\right) & =\left(\mathbf{u}_{0}, \mathbf{w}\right) & & \forall \mathbf{w} \in\left(S_{h_{n}}\right)^{2} .
\end{aligned}
$$

For computational simplicity, the nonlinear reaction term $\mathbf{f}\left(\mathbf{u}_{h}^{n}, \boldsymbol{x}_{n}\right)$ on the right-hand side of (4.5a) is linearized about the finite-element solution $\mathbf{u}_{h}^{n-1}$ at the previous time level $t_{n-1}$. The numerical method described above is similar to that given in [1] and [22]. 
5. Asymptotic and numerical results in one dimension. For the special case where $(p, q, r, s)=(2,1,2,0)$, we compare the asymptotic results presented in section 2 with corresponding numerical results. In each of the figures below, the solid curves are obtained from solving the full problem (1.1) numerically using the finiteelement method described in section 4 with approximately 1001 elements. The crosses in these figures represent the asymptotic results obtained from solving the relevant differential equation of section 2 numerically using a standard solver routine from Maple [6] and from the NAG library [26].

5.1. Experiment 1. We take the parameter values $V(x) \equiv 0, \mu(x)=1, D=1$, and $\epsilon=0.03$. The initial condition used for the finite-element solution, as obtained from $(2.3 \mathrm{c}),(2.15 \mathrm{a})$, and $(2.15 \mathrm{~b})$, is

$$
\begin{aligned}
& a(x, 0)=\frac{3 H}{2} \operatorname{sech}^{2}\left(\frac{x-x_{0}}{2 \epsilon}\right), \\
& h(x, 0)=H \frac{G\left(x ; x_{0}\right)}{G\left(x_{0} ; x_{0}\right)},
\end{aligned}
$$

where from $(2.14 \mathrm{~b})$

$$
H=\left[6 G\left(x_{0} ; x_{0}\right)\right]^{-1} .
$$

In this case, the initial condition $a(x, 0)$ consists of a spike in the activator concentration centered at $x_{0}=0.6$. The asymptotic result for the motion of the center of the spike obtained from (2.19) is

$$
\frac{d x_{0}}{d t} \sim-(0.03)^{2}\left[\tanh \left(1+x_{0}\right)-\tanh \left(1-x_{0}\right)\right],
$$

with $x_{0}(0)=0.6$. In Figure 2 and Table 1 , we show the favorable agreement between the asymptotic and numerical results for the motion of the center of the spike. For this example, the spike drifts toward the center of the interval.

5.2. Experiment 2. The parameter values here are $V(x) \equiv \frac{1}{2}\left(x-\frac{1}{4}\right)^{2}, \mu(x)=1$, $D=1$, and $\epsilon=0.03$. The initial condition used for the finite-element solution is

$$
\begin{aligned}
& a(x, 0)=\frac{3}{2} H\left(1+V\left[x_{0}\right]\right) \operatorname{sech}^{2}\left(\frac{\sqrt{1+V\left[x_{0}\right]}\left(x-x_{0}\right)}{2 \epsilon}\right), \\
& h(x, 0)=H \frac{G\left(x ; x_{0}\right)}{G\left(x_{0} ; x_{0}\right)},
\end{aligned}
$$

where

$$
H=\left[6 G\left(x_{0} ; x_{0}\right)\right]^{-1}\left[1+V\left(x_{0}\right)\right]^{-3 / 2} .
$$

As in the previous experiment, we choose the initial spike location to be at $x_{0}(0)=0.6$. In this case, the differential equation for the spike location $x_{0}(t)$, as obtained from $(2.19)$, is

$$
\frac{d x_{0}}{d t} \sim-(0.03)^{2}\left(\tanh \left(1+x_{0}\right)-\tanh \left(1-x_{0}\right)+\frac{5\left(x_{0}-\frac{1}{4}\right)}{2+\left(x_{0}-\frac{1}{4}\right)^{2}}\right),
$$




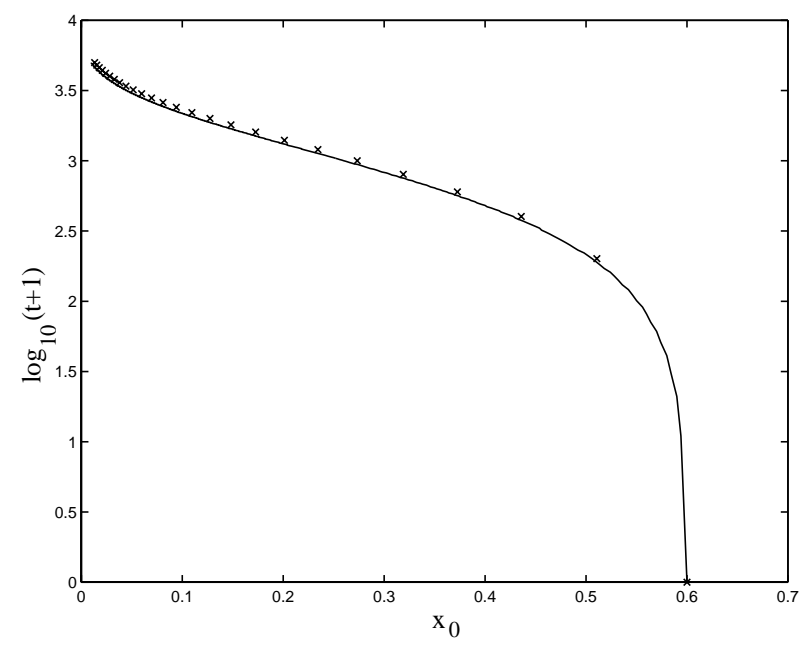

FIG. 2. The trajectory $x_{0}(t)$ of the spike in the activator concentration. The solid curve is the numerical result, and the crosses are obtained from solving the asymptotic result (5.2) numerically.

TABLE 1

The numerical and asymptotic results for $x_{0}(t)$ corresponding to a selection of the values plotted in Figure 2.

\begin{tabular}{|l|l|l|l|}
\hline \hline$t$ & $\log _{10}(t+1)$ & $x_{0}(t)(\mathrm{num})$ & $x_{0}(t)(\mathrm{asm})$ \\
\hline 0 & 0.0 & 0.6000 & 0.6 \\
10 & 1.041 & 0.5940 & 0.5951 \\
100 & 2.0043 & 0.5500 & 0.5534 \\
200 & 2.3032 & 0.5060 & 0.5107 \\
400 & 2.6031 & 0.4280 & 0.4358 \\
800 & 2.9036 & 0.3060 & 0.3189 \\
1600 & 3.2044 & 0.1600 & 0.1727 \\
3200 & 3.5053 & 0.0440 & 0.0514 \\
\hline \hline
\end{tabular}

with $x_{0}(0)=0.6$. As a result of the competition between the hyperbolic tangent functions and the precursor gradient $V(x)$, the spike does not approach the center of the interval as $t \rightarrow \infty$. Instead, by setting the right-hand side of (5.4) to zero, it follows that (5.4) has a unique stable equilibrium location $x_{0}^{e} \neq 0$ for which $\lim _{t \rightarrow \infty} x_{0}(t) \rightarrow$ $x_{0}^{e} \approx 0.184$. In Figure 3 and Table 2, we favorably compare the asymptotic and numerical results for the motion of the center of the spike.

5.3. Experiment 3. The parameter values are $V(x) \equiv \frac{1}{2}\left(x-\frac{1}{4}\right)^{2}, \mu(x)=1$, $D=50$, and $\epsilon=0.03$. The initial conditions are as in the previous experiment. In this case, the appropriate differential equation is $(2.25)$, which reduces to

$$
\frac{d x_{0}}{d t} \sim-(0.03)^{2}\left(\frac{x_{0}}{25}+\frac{5\left(x_{0}-\frac{1}{4}\right)}{2+\left(x_{0}-\frac{1}{4}\right)^{2}}\right),
$$

with $x_{0}(0)=0.6$. From this differential equation it follows that the equilibrium position of the spike is approximately $\frac{1}{4}$ since the contribution to the dynamics from the first term is negligible. Thus, in this case, the spike tends to the minimum value 


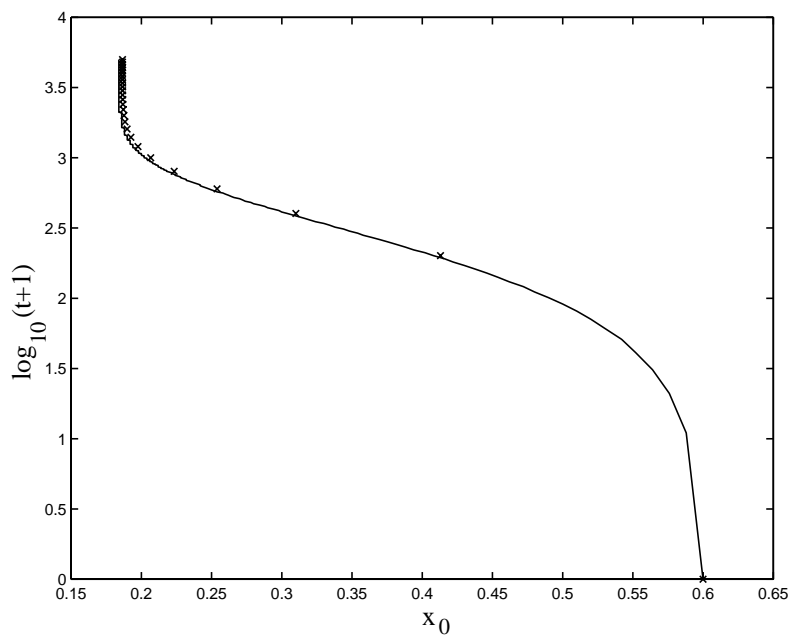

FIG. 3. The trajectory $x_{0}(t)$ of the spike in the activator concentration. The solid curve is the numerical result, and the crosses are obtained from solving the asymptotic result (5.4) numerically.

TABLE 2

The numerical and asymptotic results for $x_{0}(t)$ corresponding to a selection of the values plotted in Figure 3.

\begin{tabular}{|l|l|l|l|}
\hline \hline$t$ & $\log _{10}(t+1)$ & $x_{0}(t)(\mathrm{num})$ & $x_{0}(t)(\mathrm{asm})$ \\
\hline 0 & 0.0 & 0.6000 & 0.6 \\
10 & 1.041 & 0.5880 & 0.5879 \\
100 & 2.0043 & 0.4900 & 0.4929 \\
200 & 2.3032 & 0.4080 & 0.4130 \\
400 & 2.6031 & 0.3040 & 0.3100 \\
800 & 2.9036 & 0.2180 & 0.2234 \\
1600 & 3.2044 & 0.1880 & 0.1899 \\
3200 & 3.5053 & 0.1840 & 0.1867 \\
\hline \hline
\end{tabular}

of the potential function $V(x)$ as $t \rightarrow \infty$. The asymptotic and numerical results for the center of the spike as a function of time are compared in Figure 4 and Table 3.

5.4. Experiment 4. Here we set $V(x) \equiv 0, \mu(x)=2 e^{-(x+1)}, D=50$, and $\epsilon=0.03$. We take the same initial conditions as in experiment 1. For this example, the spike location satisfies $(2.25)$, which reduces to

$$
\frac{d x_{0}}{d t} \sim-\frac{(0.03)^{2}}{25}\left(2 \int_{-1}^{x_{0}} e^{-(x+1)} \mathrm{d} x-\mu_{a}\right),
$$

with $x_{0}(0)=0.6$. From (5.6) it follows that there is a unique stable equilibrium value $x_{0}^{e}$ satisfying

$$
\int_{-1}^{x_{0}^{e}} \mu(y) \mathrm{d} y=\mu_{a}, \quad \text { where } \quad \mu_{a}=\frac{1}{2} \int_{-1}^{1} \mu(y) \mathrm{d} y .
$$

Using $\mu(x)=2 e^{-(x+1)},(5.7)$ yields $x_{0}^{e}=-1-\log \left(1-\frac{\mu_{a}}{2}\right) \approx-0.434$, and thus $\lim _{t \rightarrow \infty} x_{0}(t) \rightarrow x_{0}^{e}$. Asymptotic and numerical results for the center of the spike as a function of time are compared in Figure 5 and Table 4. 


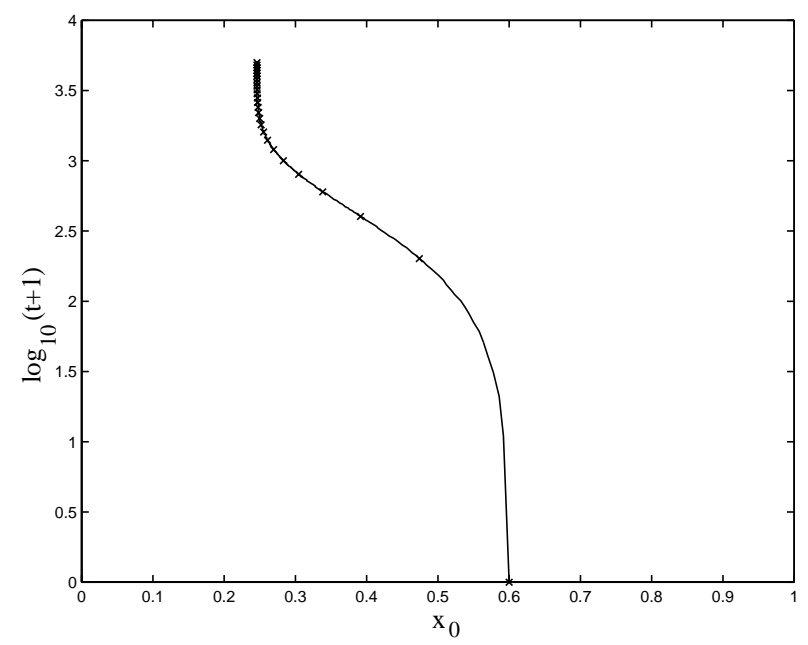

FIG. 4. The trajectory $x_{0}(t)$ of the spike in the activator concentration. The solid curve is the numerical result, and the crosses are obtained from solving the asymptotic result (5.5) numerically.

TABLE 3

The numerical and asymptotic results for $x_{0}(t)$ corresponding to a selection of the values plotted in Figure 4.

\begin{tabular}{|l|l|l|l|}
\hline \hline$t$ & $\log _{10}(t+1)$ & $x_{0}(t)(\mathrm{num})$ & $x_{0}(t)(\mathrm{asm})$ \\
\hline 0 & 0.0 & 0.6000 & 0.6 \\
10 & 1.041 & 0.5920 & 0.5924 \\
100 & 2.0043 & 0.5320 & 0.5306 \\
200 & 2.3032 & 0.4740 & 0.4740 \\
400 & 2.6031 & 0.3920 & 0.3914 \\
800 & 2.9036 & 0.3060 & 0.3045 \\
1600 & 3.2044 & 0.2560 & 0.2555 \\
3200 & 3.5053 & 0.2460 & 0.2463 \\
\hline \hline
\end{tabular}

5.5. Experiment 5. Here we take $V(x) \equiv \frac{1}{2}\left(x-\frac{1}{4}\right)^{2}, \mu(x)=2 e^{-(x+1)}, D=1.0$, and $\epsilon=0.03$. We take the same initial conditions as in experiment 2 .

For this example, the spike location satisfies the differential equation (2.17). In order to evaluate Green's function appearing in the asymptotic result (2.17), we solved the boundary value problem (2.12) numerically using COLSYS [2] for a range of $x_{0}$ values. A spline interpolant was then used to evaluate the first term on the righthand side of (2.17) at an arbitrary value of $x_{0}$. The stable equilibrium value for $(2.17)$ was found numerically to be $x_{0}^{e} \approx 0.056$. Thus we have that $\lim _{t \rightarrow \infty} x_{0}(t) \rightarrow 0.056$. Asymptotic and numerical results for the center of the spike as a function of time are compared in Figure 6 and Table 5.

6. Asymptotic and numerical results in two dimensions. For the special case where $(p, q, r, s)=(2,1,2,0)$, we compare the asymptotic results presented in section 3 with corresponding numerical results. For each of the examples below we took a circular domain of radius one. In the numerical computations shown, we took 8321 nodes and 16384 elements for the finite-element method of section 4 . The 


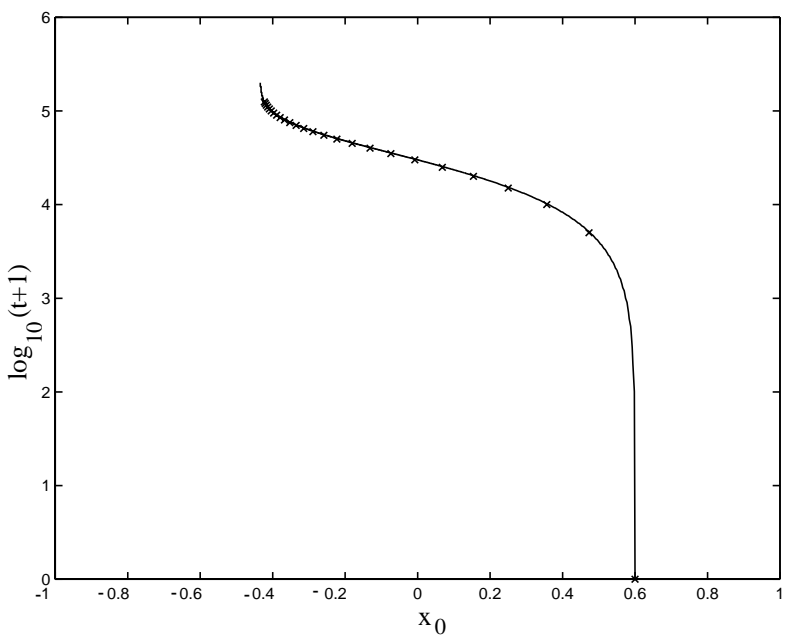

FIG. 5. The trajectory $x_{0}(t)$ of the spike in the activator concentration. The solid curve is the numerical result, and the crosses are obtained from solving the asymptotic result (5.6) numerically.

TABLE 4

The numerical and asymptotic results for $x_{0}(t)$ corresponding to a selection of the values plotted in Figure 5.

\begin{tabular}{|l|l|r|r|}
\hline \hline$t$ & $\log _{10}(t+1)$ & $x_{0}(t)(\mathrm{num})$ & $x_{0}(t)(\mathrm{asm})$ \\
\hline 0 & 0 & 0.6000 & 0.6 \\
100 & 2.0043 & 0.5980 & 0.5974 \\
200 & 2.3032 & 0.5940 & 0.5947 \\
400 & 2.6031 & 0.5900 & 0.5895 \\
800 & 2.9036 & 0.5800 & 0.5791 \\
1600 & 3.2044 & 0.5600 & 0.5584 \\
3200 & 3.5053 & 0.5200 & 0.5177 \\
6400 & 3.8062 & 0.4420 & 0.4395 \\
12800 & 4.1072 & 0.3020 & 0.2955 \\
25600 & 4.4081 & 0.0660 & 0.0748 \\
51200 & 4.7095 & -0.2260 & -0.2166 \\
102400 & 5.0104 & -0.4060 & -0.4018 \\
\hline \hline
\end{tabular}

circular domain was triangulated in a manner similar to that shown in Figure 7. A fixed time step, $\Delta t=0.1$, was chosen in the simulations. The general form of the initial conditions used in the simulations is

$$
\begin{aligned}
& a(\boldsymbol{x}, 0)=\sum_{i=1}^{m} \operatorname{sech}^{8}\left[\varepsilon^{-1}\left|\boldsymbol{x}-\boldsymbol{x}_{\boldsymbol{i}}\right|\right] \\
& h(\boldsymbol{x}, 0)=100.0
\end{aligned}
$$

where $\boldsymbol{x}_{\boldsymbol{i}}$ is the location of the $i$ th spike and $m$ is the number of spikes.

6.1. Experiment 1: The effect of $\boldsymbol{D}$ when $\boldsymbol{V} \equiv \mathbf{0}$. We set $V(x) \equiv 0$ and $\mu(x)=1$. The initial condition for $a$ in $(6.1)$ is taken to consist of a single spike in the activator concentration centered at $(-0.5,-0.5)$. The asymptotic result for the 


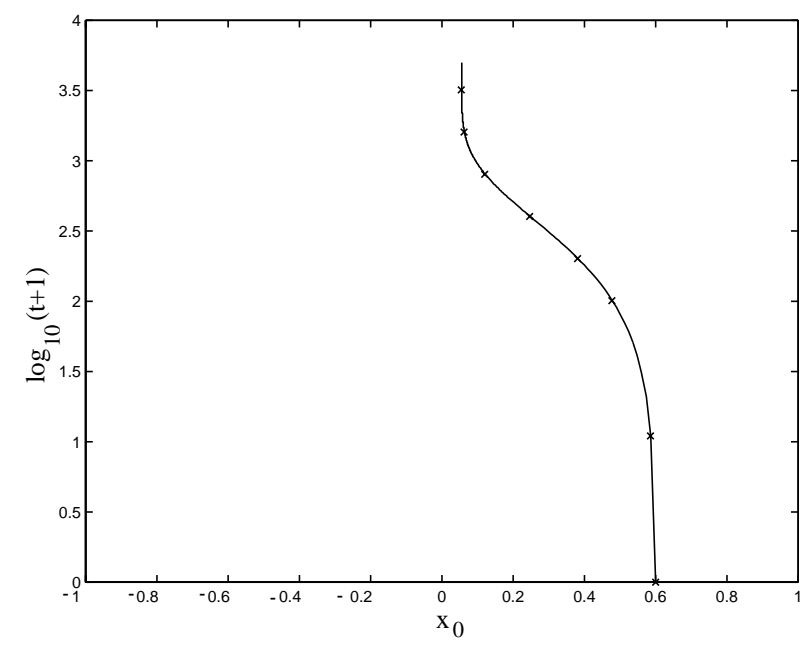

FIG. 6. The trajectory $x_{0}(t)$ of the spike in the activator concentration. The solid curve is the numerical result, and the crosses are obtained from solving the asymptotic result (2.17) numerically.

TABLE 5

The numerical and asymptotic results for $x_{0}(t)$ corresponding to the values plotted in Figure 6.

\begin{tabular}{|l|l|r|r|}
\hline \hline$t$ & $\log _{10}(t+1)$ & $x_{0}(t)(\mathrm{num})$ & $x_{0}(t)(\mathrm{asm})$ \\
\hline 0 & 0.0 & 0.6000 & 0.6 \\
10 & 1.041 & 0.5860 & 0.5854 \\
100 & 2.0043 & 0.4780 & 0.4771 \\
200 & 2.3032 & 0.3820 & 0.3808 \\
400 & 2.6031 & 0.2480 & 0.2464 \\
800 & 2.9036 & 0.1220 & 0.1200 \\
1600 & 3.2044 & 0.0640 & 0.0621 \\
3200 & 3.5053 & 0.0560 & 0.0557 \\
\hline \hline
\end{tabular}

distance of the spike to the origin is given in (3.50), and it is valid when $\varepsilon \ll 1$ and $D \gg 1$. In Figure $8 \mathrm{a}$, where $D=10$ and $\varepsilon=0.06$, we show the close agreement between the full numerical result for the distance of the spike to the origin and the asymptotic result obtained from (3.50). The asymptotic result (3.50) is no longer valid when $D=O(1)$ since the leading order problem (3.31) is strongly coupled, which makes the analysis more difficult. In Figure $8 \mathrm{~b}$, where $D=1$ and $\varepsilon=0.03$, we show the rather poor agreement between the full numerical result for the distance of the spike to the origin and the result obtained from (3.50). The motion of a spike for the case $D=O(1)$ is similar to that shown in Figures 11c and 11d below. It would be interesting to analytically characterize the one-spike dynamics when $D=O(1)$. We remark that a limitation of the numerical method used to generate Figures $8 \mathrm{a}$ and $8 \mathrm{~b}$ is that they were done with a small fixed time step. To improve accuracy and minimize computational costs over a long time interval, it would be preferable to implement a variable time step strategy with error control.

6.2. Experiment 2: The effect of the potential $\boldsymbol{V}$. We take $V(\boldsymbol{x}) \equiv \frac{1}{4} \mid \boldsymbol{x}-$ $\left.\xi\right|^{2}$, where $\xi=(0.25,-0.25)$, so that the potential $W$ in $(3.27)$ has a minimum at 


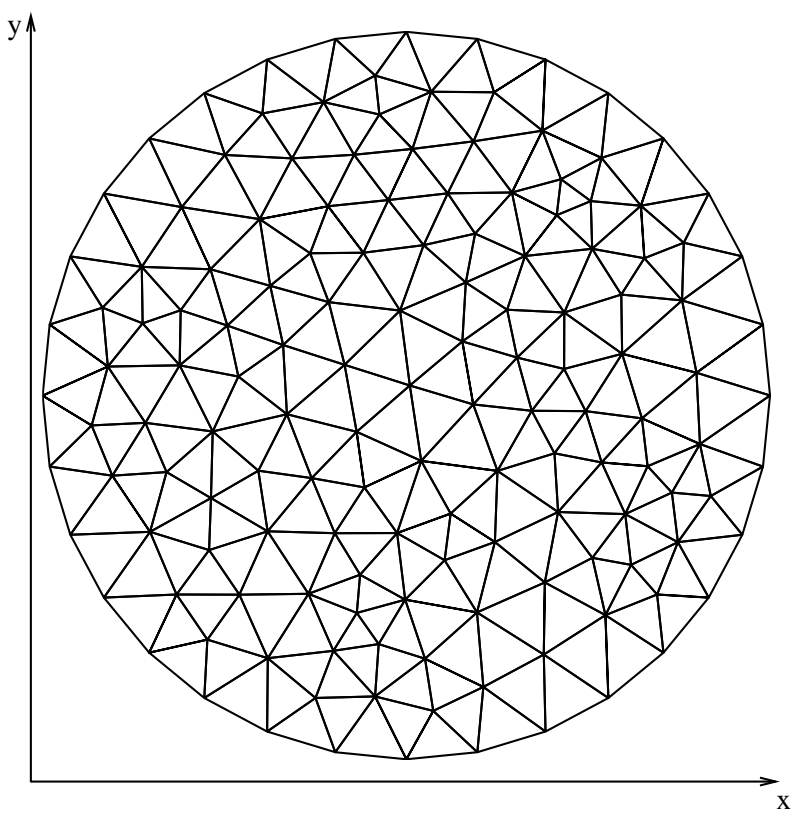

FIG. 7. A regular triangulation of a circular domain.

$\boldsymbol{x}=\xi$. From (3.27) we expect that $\boldsymbol{x} \rightarrow \xi$ as $t \rightarrow \infty$. The other parameter values are $D=25, \mu=1$, and $\varepsilon=0.10$. Since $D \gg 1$, the asymptotic result for the spike motion is (3.27). The initial condition in $a$, given in (6.1a), is taken to be a single spike centered at $\mathbf{x}_{\mathbf{0}}(0)=(-0.5,-0.5)$. From the asymptotic result $(3.27)$, we can derive the following system for the coordinates $\boldsymbol{x}_{\mathbf{0}}=\left(x_{0}, y_{0}\right)$ of the center of the spike:

$$
\frac{d x_{0}}{d t} \sim-\frac{4 \varepsilon^{2}\left(x_{0}-\xi_{1}\right)}{4+\left|\boldsymbol{x}_{\mathbf{0}}-\xi\right|}, \quad \frac{d y_{0}}{d t} \sim-\frac{4 \varepsilon^{2}\left(y_{0}-\xi_{2}\right)}{4+\left|\boldsymbol{x}_{\mathbf{0}}-\xi\right|} .
$$

Here $\xi=\left(\xi_{1}, \xi_{2}\right)$. In Figure 9a, we show the close agreement between the full numerical result for the distance of the spike to the origin and the corresponding asymptotic result obtained from integrating (6.2) numerically using Maple [6]. In Figure 9b, we show that the trajectory of the spike is approximately orthogonal to the level curves of the potential $W$ as predicted by (3.27).

7. Discussion. In a one-dimensional domain, we have given a complete characterization of the dynamics of a one-spike solution to (1.1), allowing for spatially inhomogeneous precursor gradients $\mu$ and $V$. These gradients had the effect of localizing a spike to certain points in the domain, depending on certain global properties of $\mu$ and local properties of $V$. The dynamical results obtained from our asymptotic analysis have been favorably compared with full numerical simulations. We have restricted our analysis to the special case of a single spike since the analysis of multispike solutions under the effect of precursor gradients is expected to be significantly more intricate. It would be interesting to extend the analysis to the multispike case.

The analysis presented above is for the simplified forms (1.1) and (1.3) of the GM model, where the dynamical behavior of the inhibitor field is neglected. This simplification has led to a parabolic-elliptic system for $a$ and $h$. An interesting but 


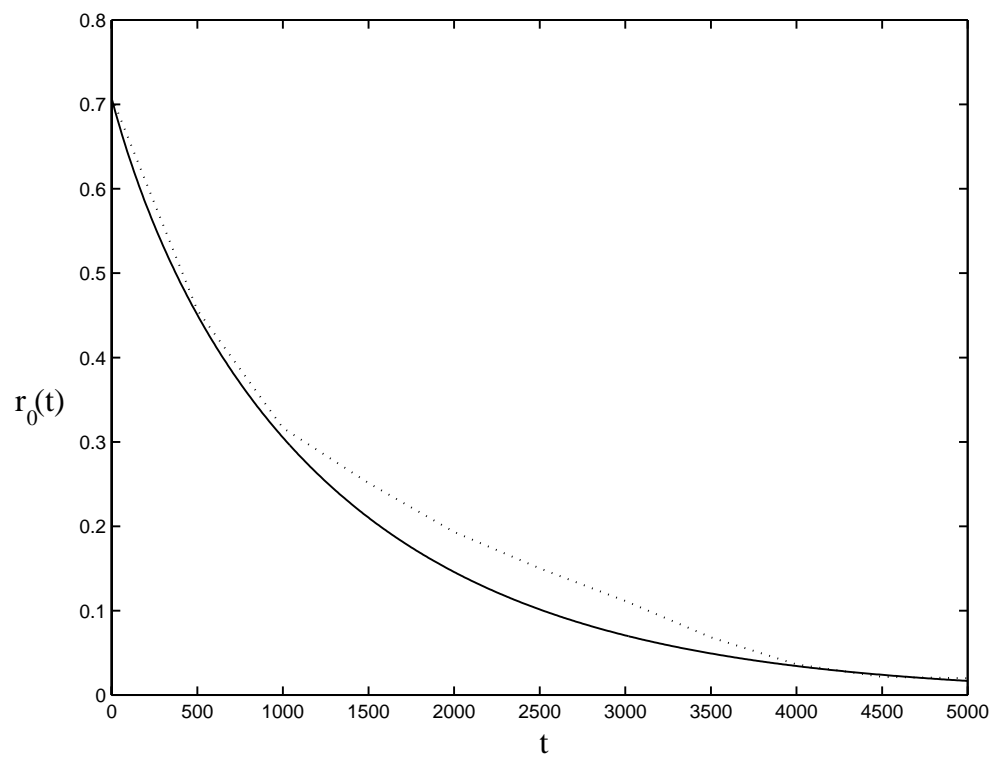

FIG. 8A. The distance from the center of the spike to the origin for experiment 1 when $D=10$ and $\varepsilon=0.06$. The dotted curve is the full numerical result, and the solid curve is the asymptotic result (3.50).

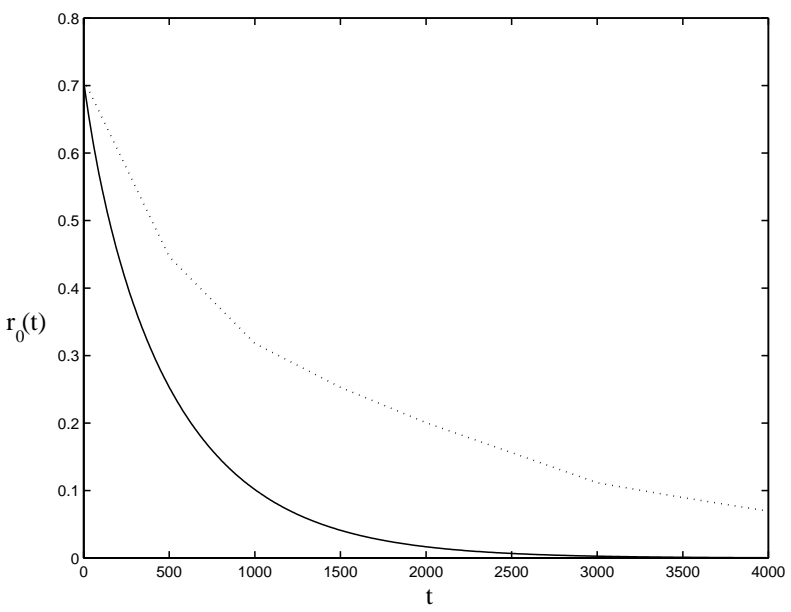

FIG. 8B. The distance from the center of the spike to the origin for experiment 1 when $D=1$ and $\varepsilon=0.03$. The dotted curve is the full numerical result, and the solid curve is the asymptotic result (3.50).

significantly more difficult problem would be to extend the analysis presented above to allow for a genuine parabolic system for $a$ and $h$ such as that obtained by replacing the left-hand side of (1.1b) and (1.3b) by $\tau_{0} h_{t}$. We expect that the effect of this term would be to introduce the possibility of Hopf bifurcations and oscillatory phenomena for certain ranges of the time constant $\tau_{0}$. This study would allow us to make quantitative comparisons of the analytical theory with the full numerical simulations of spike localization phenomena computed in [14] and [15]. 


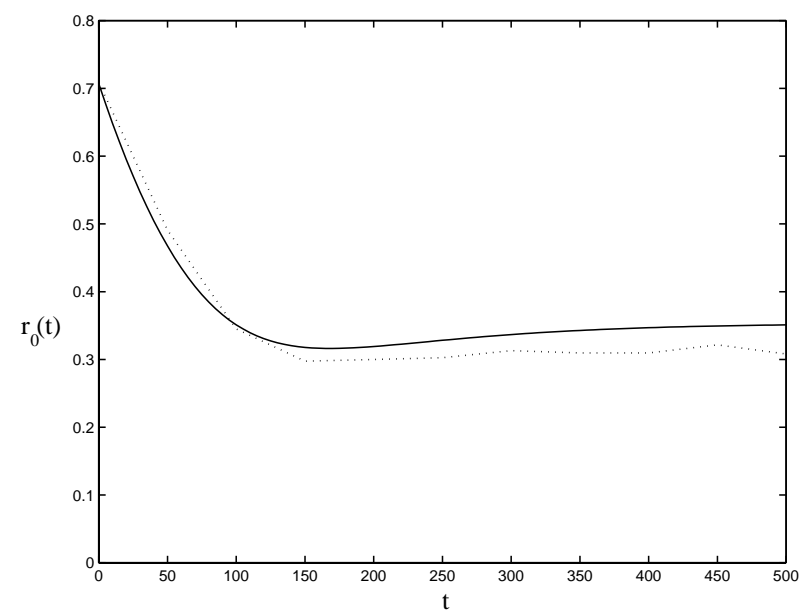

FIG. 9A. The distance from the center of the spike to the origin for experiment 2 . The dotted curve is the full numerical result, and the solid curve is the asymptotic result (6.2).

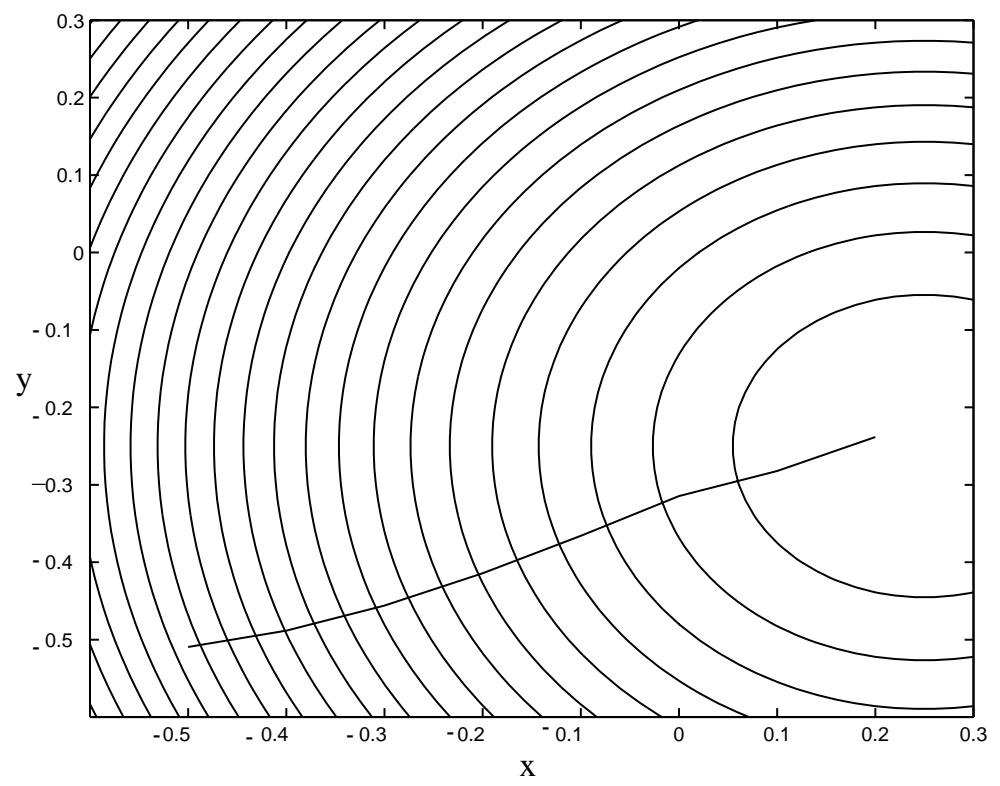

FIG. 9B. The heavy solid line is the spike trajectory from the full numerical result for experiment 2 , and the solid lines are the level curves of the potential $W$ as defined in (3.27).

Other reaction-diffusion systems also exhibit spike-type behavior, including the Gray-Scott model studied in [29], [8], and [9]. These previous studies have focused on the one-dimensional problem in the absence of precursor gradients. It would be interesting to extend their results on spike dynamics and spike replication to allow for the effect of precursor gradients.

For the two-dimensional problem (1.3), under the assumption that $D \gg-\log \varepsilon$, we studied the evolution of a one-spike solution allowing for spatially inhomogeneous precursor gradients in both $V$ and $\mu$. Using a finite-element solution, we compared 
our asymptotic results in the case of a circular domain for two different forms of $V$ when $\mu=1$. The evolution of a one-spike solution when $D=O(1)$ will require further study since in this case the activator and inhibitor fields are strongly coupled in the core of the spike. This will lead to a different law of motion from what we have derived.

Most of the full numerical simulations of (1.3) (e.g., [14] and [25]) involve the motion of several or even many spikes inside the domain. We now give a glimpse at the behavior of a two-spike solution with spatially homogeneous coefficients. In this experiment, we examine numerically the behavior of the solution to (1.3) at two different values of $D$ when the initial condition in $a$ consists of two spikes centered at $(-0.5,0.0)$ and $(0.5,0.0)$. The initial profiles for $a$ and $h$ are given by (6.1). The parameter values are $V(x) \equiv 0, \mu(x) \equiv 1$, and $\varepsilon=0.03$, and the domain is a unit circle. We first take $D=0.5$. For this value of $D$, each spike tends to a certain equilibrium location inside the unit circle as shown in Figures 10a and 10b. Next, for the case when $D=1.0$, we show the computational results at various times in Figures 11a-11d. For this value of $D$, we observe that one of the spikes is annihilated rather quickly, while the remaining spike drifts very slowly toward the center of the circle. This slow drift toward the origin is described by the result in Corollary 3.4.

The stability of multispike solutions in a multidimensional domain is a difficult problem. In [33], the first criterion for the stability of an $N$-spike equilibrium solution in a two-dimensional domain was derived in the limit $\varepsilon \rightarrow 0$ for the case of no pinning, where $V(x) \equiv 0$ and $\mu \equiv 1$. For $\varepsilon \rightarrow 0$, it was proven in [33] that an $N$-spike equilibrium solution is stable on an $O(1)$ time scale if and only if

$$
D<D_{N} \sim-\frac{\log \varepsilon}{2 \pi N}
$$

This result predicts that the threshold value $D_{N}$ is independent of the locations of the centers of the spikes. For the example above, where $N=2$ and $\varepsilon=0.03$, we calculate from (7.1) that $D_{2}=0.28$. However, the numerical experiment above indicates that the two-spike equilibrium solution is stable when $D=0.5>D_{2}$. We believe that this paradox results from obtaining only a leading order asymptotic expansion for $D_{N}$ in powers of $-\log \varepsilon$. We conjecture that $D_{N}$ has an infinite logarithmic expansion of the form

$$
D_{N} \sim \frac{-\log \varepsilon+F\left(\mathbf{x}_{\mathbf{i}} ; \nu(\varepsilon)\right)}{2 \pi N},
$$

where $F$ is $O(1)$ as $\varepsilon \rightarrow 0$, which can be expanded in powers of $\nu \equiv-1 / \log \varepsilon$, and depends on the equilibrium spike locations $\mathbf{x}_{i}$ for $i=1, \ldots, N$. Infinite logarithmic series, and the difficulty associated with low order truncations of these series, have been identified previously in many problems, including singularly perturbed eigenvalue problems (see [32]). We were unsuccessful in verifying the criterion (7.1) by computing full numerical solutions of (1.3) for values of $\varepsilon$ significantly smaller than $\varepsilon=0.03$. This is a result of numerical difficulties associated with having to generate a sufficiently fine finite-element mesh that can resolve the spikes for very small values of $\varepsilon$ and then having to remesh the domain as the spikes move very slowly in time. It would be very interesting to develop a theory giving a complete characterization of the stability and dynamics of multispike solutions in two dimensions with and without the effect of spatially inhomogeneous precursor gradients. 


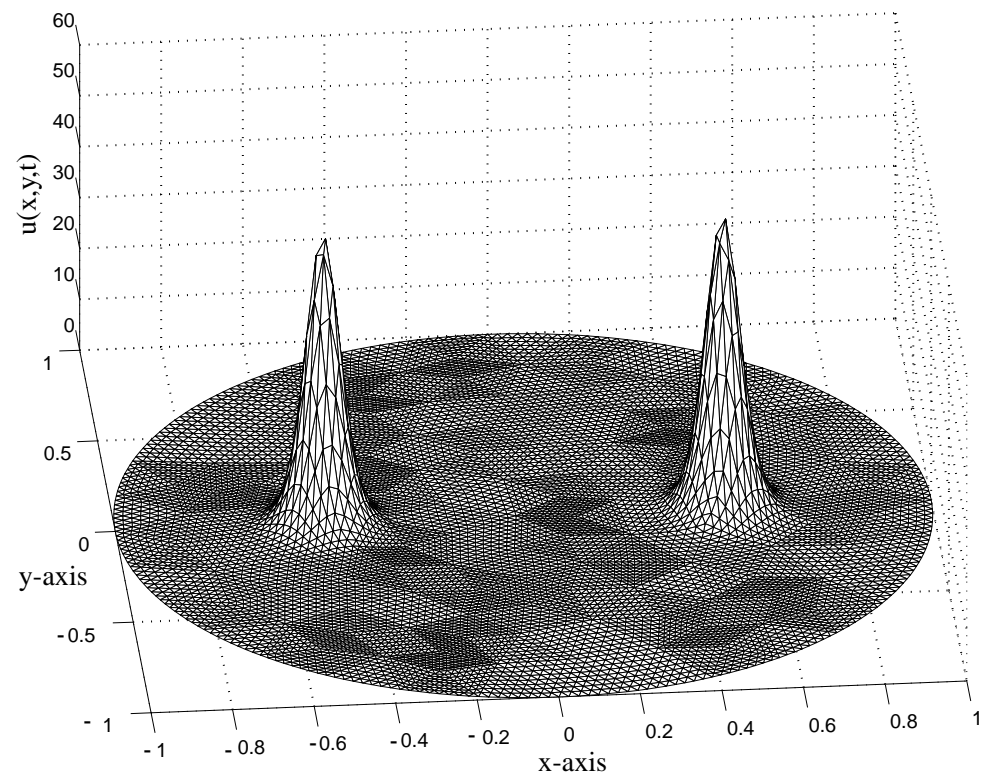

FIG. 10A. The numerical solution of $a(\boldsymbol{x}, t)$ at $t=100.0$ with parameters $\epsilon=0.03, \mu=1.0$, and $D=0.5$.

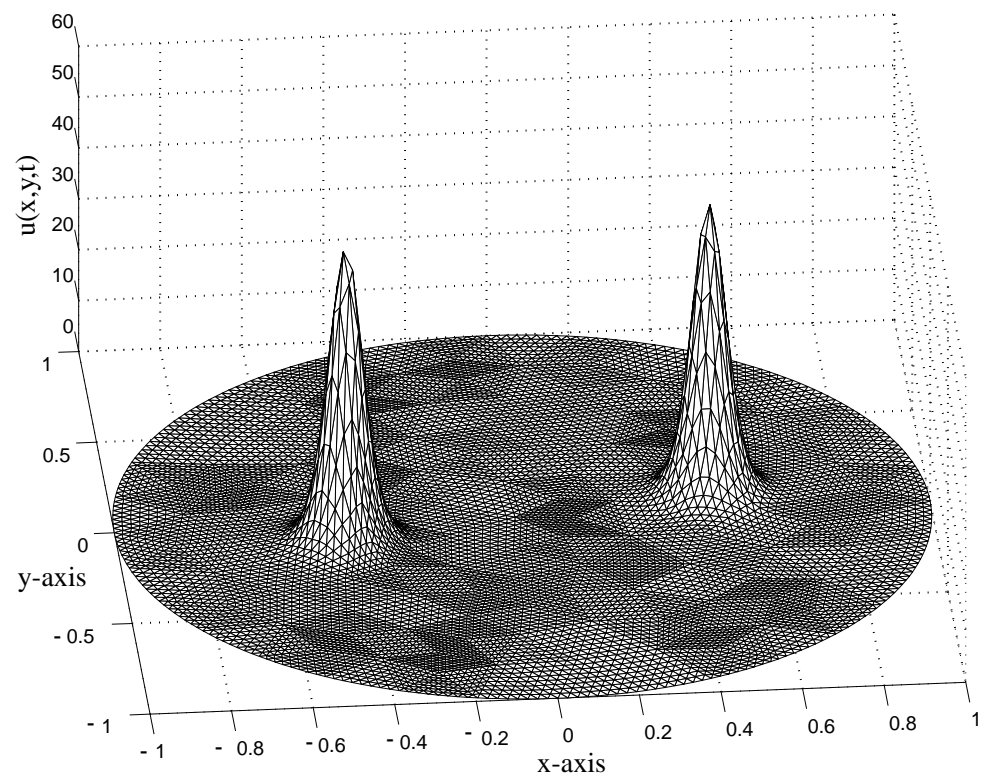

FIG. 10B. The numerical solution of $a(\boldsymbol{x}, t)$ at $t=3000.0$ with parameters $\epsilon=0.03, \mu=1.0$, and $D=0.5$. 


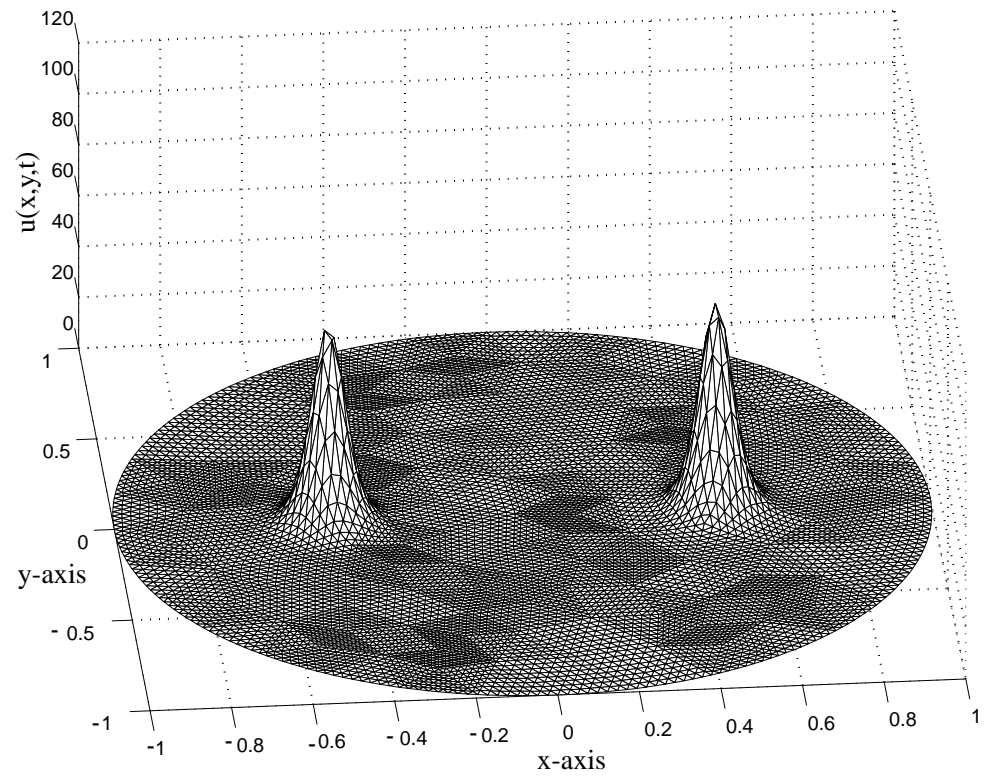

FIG. 11A. The numerical solution of $a(\boldsymbol{x}, t)$ at $t=10.0$ with parameters $\epsilon=0.03, \mu=1.0$, and $D=1.0$.

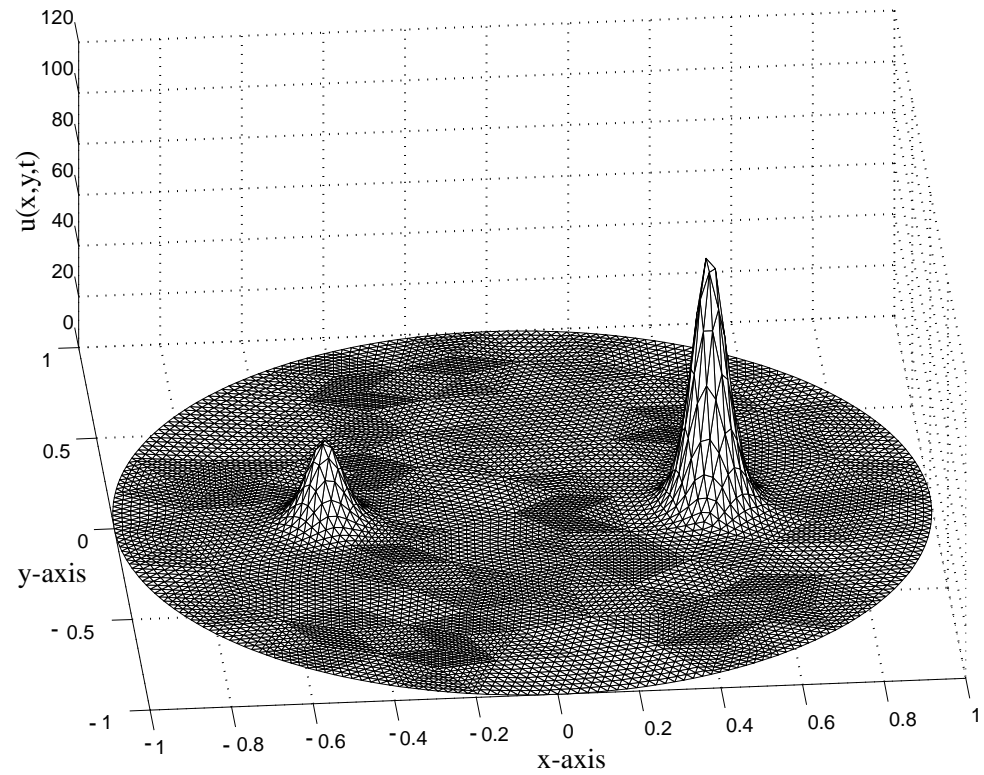

FIG. 11B. The numerical solution of $a(\boldsymbol{x}, t)$ at $t=160.0$ with parameters $\epsilon=0.03, \mu=1.0$, and $D=1.0$. 


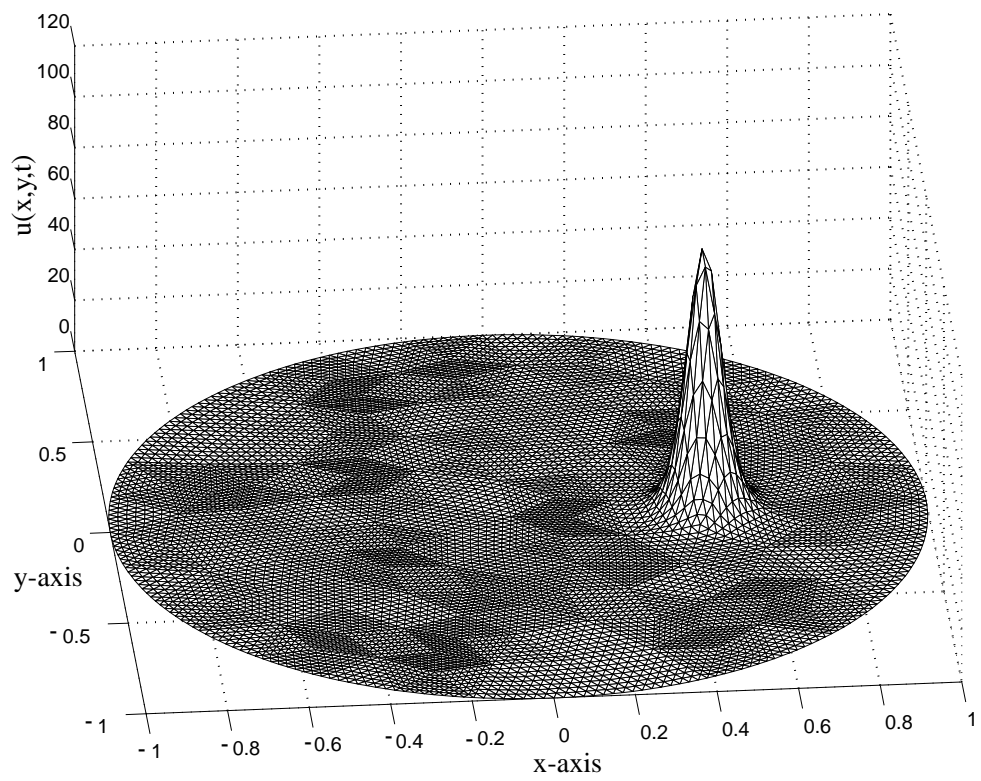

FIG. 11C. The numerical solution of $a(\boldsymbol{x}, t)$ at $t=170.0$ with parameters $\epsilon=0.03, \mu=1.0$, and $D=1.0$.

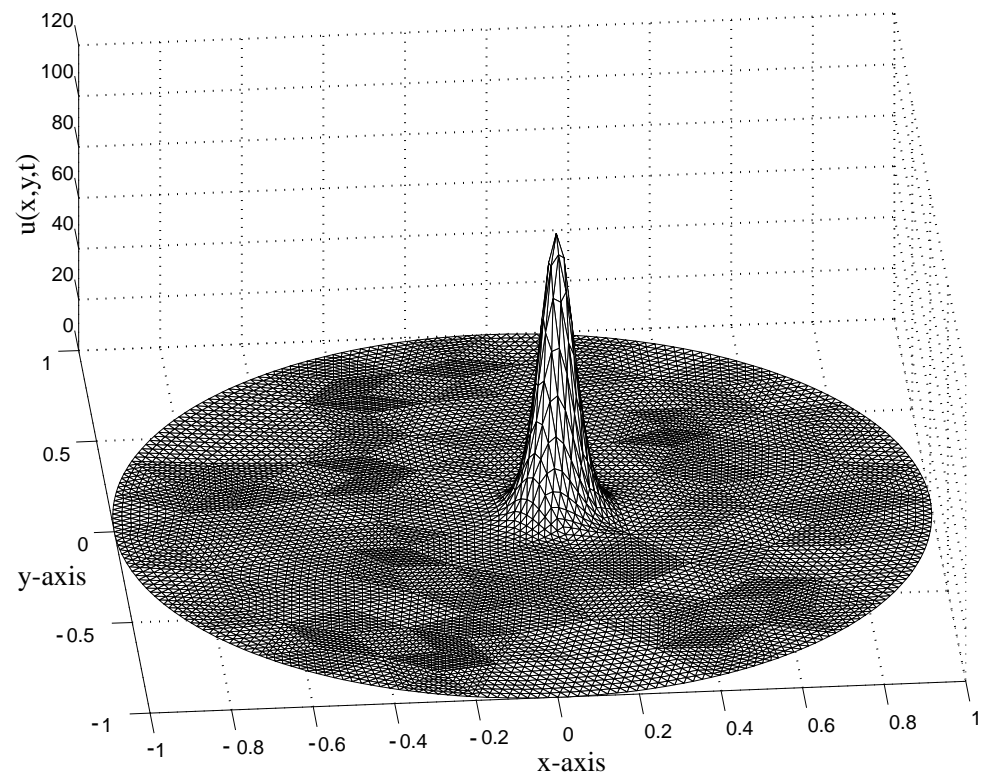

FIG. 11D. The numerical solution of $a(\boldsymbol{x}, t)$ at $t=3000.0$ with parameters $\epsilon=0.03, \mu=1.0$, and $D=1.0$. 
Appendix A. Calculating some integrals in section 2. Here we show how to calculate the integrals appearing in $(2.15 \mathrm{c})$. We define them as

$$
e \equiv \frac{\int_{-\infty}^{\infty}\left[u_{c}(y)\right]^{2} d y}{\int_{-\infty}^{\infty}\left[u_{c}^{\prime}(y)\right]^{2} d y}, \quad f \equiv \frac{\int_{-\infty}^{\infty}\left[u_{c}(y)\right]^{p+1} d y}{\int_{-\infty}^{\infty}\left[u_{c}^{\prime}(y)\right]^{2} d y} .
$$

We first multiply $(2.3 \mathrm{a})$ by $u_{c}$. Upon integrating the resulting equation over the domain, we obtain

$$
\int_{-\infty}^{\infty} u_{c} u_{c}^{\prime \prime} d y-\left[1+V\left(x_{0}\right)\right] \int_{-\infty}^{\infty} u_{c}^{2} d y+\int_{-\infty}^{\infty} u_{c}^{p+1} d y=0 .
$$

Upon integrating the first term in this equation by parts, we get

$$
-1=\left[1+V\left(x_{0}\right)\right] e-f .
$$

To obtain an additional equation, we multiply (2.3a) by $u_{c}^{\prime}$ and integrate over the domain to fix the constant of integration. We then integrate the resulting expression again to get

$$
1=\left[1+V\left(x_{0}\right)\right] e-\frac{2 f}{p+1} .
$$

Solving (A.3) and (A.4), we get the key results

$$
f=\frac{2(p+1)}{p-1}, \quad e=\frac{1}{1+V\left(x_{0}\right)}\left(\frac{p+3}{p-1}\right) .
$$

Appendix B. Calculating some integrals in section 3. We define the integrals in (3.26) and (3.39) by $b$ and $f$, respectively. They are given by

$$
b \equiv \frac{\int_{0}^{\infty}\left[w_{c}(\eta)\right]^{2} \eta d \eta}{\int_{0}^{\infty}\left[w_{c}^{\prime}(\eta)\right]^{2} \eta d \eta}, \quad f \equiv \frac{\int_{0}^{\infty}\left[w_{c}(\eta)\right]^{p+1} \eta d \eta}{\int_{0}^{\infty}\left[w_{c}^{\prime}(\eta)\right]^{2} \eta d \eta} .
$$

We show how to calculate them in terms of the solution $w_{c}$ to (3.24).

We first multiply (3.24a) by $\eta w_{c}$. Upon integrating the resulting equation over the domain, we obtain

$$
\int_{0}^{\infty} w_{c}\left(\eta w_{c}^{\prime}\right)^{\prime} d \eta-\int_{0}^{\infty} \eta w_{c}^{2} d \eta+\int_{0}^{\infty} \eta w_{c}^{p+1} d \eta=0 .
$$

Upon integrating the first term in this equation by parts, we get

$$
f=b+1 .
$$

To obtain an additional equation, we multiply (3.24a) by $\eta^{2} w_{c}^{\prime}$ and integrate over the domain. Upon integrating by parts and using the decay of $w_{c}$ at infinity, we obtain

$$
b=2 f /(p+1) .
$$

Solving (B.3) and (B.4), we get the key results

$$
f=\frac{p+1}{p-1}, \quad b=\frac{2}{p-1} .
$$


Acknowledgments. M. J. Ward is grateful for the hospitality of Hilary and John Ockendon of OCIAM at Oxford University, where this paper was initiated.

\section{REFERENCES}

[1] G. Akrivis, M. Crouzeix, and C. Makridakis, Implicit-explicit multistep finite element methods for nonlinear parabolic problems, Math. Comp., 67 (1998), pp. 457-477.

[2] U. Ascher, R. Christiansen, and R. Russell, A collocation solver for mixed order systems of boundary value problems, Math. Comp., 33 (1979), pp. 659-679.

[3] M. Booty and G. Kriegsmann, Microwave heating and joining of ceramic cylinders: A mathematical model, Methods Appl. Anal., 4 (1994), pp. 403-414.

[4] A. Bose And G. Kriegsmann, Large amplitude solutions of spatially non-homogeneous nonlocal reaction-diffusion equations, Methods Appl. Anal., 7 (2000), pp. 295-312.

[5] S. J. Chapman and G. Richardson, Vortex pinning by inhomogeneities in type-2 superconductors, Phys. D, 108 (1997), pp. 397-407.

[6] B. W. Char, K. O. Geddes, G. H. Gonnet, B. L. Leong, M. B. Monagan, and S. M. WatT, Maple V Language Reference Manual, Springer-Verlag, New York, 1991.

[7] X. Chen And M. Kowalcyzk, Dynamics of an interior spike in the Gierer-Meinhardt system, SIAM J. Math. Anal., 33 (2001), pp. 172-193.

[8] A. Doelman, T. J. Kaper, and P. Zegeling, Pattern formation in the one-dimensional Gray-Scott model, Nonlinearity, 10 (1997), pp. 523-563.

[9] A. Doelman, R. A. Gardner, And T. J. Kaper, Stability analysis of singular patterns in the 1-D Gray-Scott model: A matched asymptotics approach, Phys. D, 122 (1998), pp. 1-36.

[10] A. Gierer and H. Meinhardt, A theory of biological pattern formation, Kybernetik, 12 (1972), pp. 30-39.

[11] C. GUI, Existence of multi-bump solutions for nonlinear Schrödinger equations via variational method, Comm. Partial Differential Equations, 5-6 (1996), pp. 787-820.

[12] J. Hale And K. SaKamoto, Existence and stability of transition layers, Japan J. Appl. Math., 5 (1988), pp. 367-405.

[13] J. K. Hale and J. D. Salazar GonzÁlez, Attractors of some reaction diffusion problems, SIAM J. Math. Anal., 30 (1999), pp. 963-984.

[14] L. HARRISON AND D. Holloway, Order and localization in reaction-diffusion pattern, Phys. A, 222 (1995), pp. 210-233.

[15] D. Holloway, L. Harrison, And J. Armstrong, Computations of post-inductive dynamics in Axolotl heart formation, Develop. Dynam., 200 (1994), pp. 242-256.

[16] D. Holloway, Reaction-Diffusion Theory of Localized Structures with Applications to Vertebrate Organogenesis, Ph.D. thesis, Department of Chemistry, University of British Columbia, Vancouver, Canada, 1995.

[17] A. Hunding, Bifurcations in Turing systems of the second kind may explain blastula cleavage plane orientation, J. Math. Biol., 25 (1987), pp. 109-121.

[18] A. Hunding, Morphogen prepatterns during mitosis and cytokinesis in flattened cells: Threedimensional Turing structures of reaction-diffusion systems in cylindrical coordinates, J. Theoret. Biol., 114 (1985), pp. 571-588.

[19] D. Iron AND M. J. WARD, A metastable spike solution for a nonlocal reaction-diffusion model, SIAM J. Appl. Math., 60 (2000), pp. 778-802.

[20] D. IRON AND M. J. WARD, The dynamics of multi-spike solutions to the one-dimensional Gierer-Meinhardt model, SIAM J. Appl. Math., submitted, 2001.

[21] D. Iron, M. J. WARD, AND J. WeI, The stability of spike solutions to the one-dimensional Gierer-Meinhardt model, Phys. D, 150 (2001), pp. 25-62.

[22] S. Larsson, Numerical analysis of semilinear parabolic problems, in The Graduate Student's Guide to Numerical Analysis '98, Springer Ser. Comput. Math. 26, M. Ainsworth, J. Levesley, and M. Marletta, eds., Springer-Verlag, Berlin, 1999, pp. 83-117.

[23] F. H. Lin AND Q. Du, Ginzburg-Landau vortices: Dynamics, pinning, and hysteresis, SIAM J. Math. Anal., 28 (1997), pp. 1265-1293.

[24] P. K. Maini, K. J. Painter, and H. N. P. Chau, Spatial pattern formation in chemical and biological systems, Faraday Trans., 93 (1997), pp. 3601-3610.

[25] H. Meinhardt, Models of Biological Pattern Formation, Academic Press, London, 1982.

[26] Numerical Algorithms Group, NAG Fortran Library Mark 17, Routine D03PCF, Oxford, UK, 1995.

[27] N. N. NefEdov, Nonstationary contrast structures of spike type in nonlinear singularly perturbed parabolic equations, Russian Acad. Sci. Dokl. Math., 49 (1994), pp. 489-492. 
[28] W. NI, Diffusion, cross-diffusion, and their spike-layer steady-states, Notices Amer. Math. Soc., 45 (1998), pp. 9-18.

[29] W. N. Reynolds, J. Pearson, and S. Ponce-Dawson, Self-replicating spots in reactiondiffusion systems, Phys. Rev. E (3), 56 (1997), pp. 185-198.

[30] I. Takagi, Point-condensation for a reaction-diffusion system, J. Differential Equations, 61 (1986), pp. 208-249.

[31] A. Turing, The chemical basis of morphogenesis, Philos. Trans Roy. Soc. B, 237 (1952), pp. $37-72$.

[32] M. J. Ward, W. D. Henshaw, and J. B. Keller, Summing logarithmic expansions for singularly perturbed eigenvalue problems, SIAM J. Appl. Math. 53 (1993), pp. 799-828.

[33] J. Wei And M. Winter, Spikes for the two-dimensional Gierer-Meinhardt system: The weak coupling case, J. Nonlinear Sci., to appear. 